29 October 2020

\title{
Sodium channel toxin-resistance mutations do not govern batrachotoxin (BTX) autoresistance in poison birds and frogs
}

Fayal Abderemane-Ali ${ }^{1}$, Nathan D. Rossen ${ }^{1}$, Megan E. Kobiela ${ }^{2}$, Robert A. Craig $\mathrm{II}^{3}$, Catherine E. Garrison $^{3}$, Lauren A. O'Connell ${ }^{4}$, J. Du Bois ${ }^{3}$, John P. Dumbacher ${ }^{5,6}$, and Daniel L. Minor, Jr. ${ }^{1,7-10^{*}}$

${ }^{1}$ Cardiovascular Research Institute

${ }^{7}$ Departments of Biochemistry and Biophysics, and Cellular and Molecular Pharmacology

${ }^{8}$ California Institute for Quantitative Biomedical Research

${ }^{9}$ Kavli Institute for Fundamental Neuroscience

University of California, San Francisco, California 93858-2330 USA

${ }^{10}$ Molecular Biophysics and Integrated Bio-imaging Division

Lawrence Berkeley National Laboratory, Berkeley, CA 94720 USA

${ }^{2}$ School of Biological Sciences, University of Nebraska - Lincoln, Lincoln, Nebraska 68588

${ }^{3}$ Department of Chemistry

Stanford University, Stanford, CA 94305

${ }^{4}$ Department of Biology

Stanford University, Stanford, CA 94305

${ }^{5}$ Institute for Biodiversity Science and Sustainability

California Academy of Sciences, San Francisco, CA 94118, USA

${ }^{6}$ Department of Biology, San Francisco State University, San Francisco, CA 94132, USA

*Correspondence to: daniel.minor@ucsf.edu

Short Title: Origin of poison bird and frog BTX resistance 


\begin{abstract}
Poisonous organisms carry small molecule toxins that alter voltage-gated sodium channel (Nav) function. Among these, batrachotoxin (BTX) from Pitohui toxic birds and Phyllobates poison frogs, stands out because of its lethality and unusual effects on Nav function. How these toxin-bearing organisms avoid autointoxication remains poorly understood. In poison frogs, a Nav DIVS6 pore-forming helix $\mathrm{N} \rightarrow \mathrm{T}$ mutation has been proposed as the BTX resistance mechanism. Here, we show that this variant is absent from Pitohui and poison frog Navs, incurs a strong cost that compromises channel function, and fails to produce BTX-resistant channels when tested in the context of poison frog Navs. We further show that captive-raised poison frogs are BTX resistant, even though they bear BTX-sensitive Navs. Hence, our data refute the hypothesis that BTX autoresistance is rooted in $\mathrm{Na}_{v}$ mutations and instead suggest that more generalizable mechanisms such as toxin sequestration act to protect BTX-bearing species from autointoxication.
\end{abstract}




\section{Introduction}

Many organisms harbor small molecule toxins that target ion channels as a means of defense from predation (1). Among these toxins, batrachotoxin (BTX) is a renowned steroidal amine that is found in distantly related vertebrate lineages including neotropical poison frogs (Dendrobatidae) (2) and multiple poisonous bird species (Pitohui sp. and Ifrita kowaldi) (3, 4). These animals acquire BTX from their diet (5-7) and accumulate it in their muscles as well as in their skin and feathers where this toxin is used as a predation defense $(2,8)$. BTX has an unusual mechanism of action against voltage-gated sodium channels ( $\mathrm{Navs)}$ that facilitates channel opening and prevents channel inactivation (9-12). How vertebrates that bear BTX or other small molecule toxins avoid autointoxication remains unresolved (13-15). Toxin-resistant mutants of target ion channels in the host organisms (16-19) or their predators (20-22) have been suggested as the primary driver of toxin autoresistance (2). Phyllobates terribilis, the poison frog carrying the highest BTX levels, is BTX-resistant, even when animals are captive-raised and lack toxin (23), suggesting that BTX resistance is a heritable trait. Partial sequencing of poison frog Navs has suggested a number of Nav mutations that might alter BTX sensitivity (24). One of these, a conserved Domain IV S6 helix (DIVS6) $\mathrm{N} \rightarrow$ T variation in P. terribilis skeletal muscle Nav1.4, reduces BTX sensitivity when tested in rat Nav1.4 (25). This result has been used to support the notion that BTX-resistance relies on toxin-resistant channel mutants, similar to examples for tetrodotoxin (TTX) (18-22) and saxitoxin (STX) (17) resistant Navs, and epibatidine-resistant nicotinic acetylcholine receptors (nAChRs) (16). However, this DIVS6 $\mathrm{N} \rightarrow \mathrm{T}$ change occurs with very low frequency among wild $P$. terribilis (26) and is absent in wild Phyllobates aurotaenia (24, 26), another poison frog species known to carry high BTX levels (27). Further, in the wild there are a number of examples of poisonous frogs $(24,26)$ and predators of toxic animals $(28)$ that lack toxin-resistant mutations, raising questions about generality of the target-based mechanism. Given these issues and the fact that no functional studies of poison frog Navs have been published, whether BTX-bearing poison animals rely on Nav mutations or some other mechanism for BTX autoresistance remains unresolved.

Here, we cloned and characterized Navs from the BTX-bearing bird Pitohui uropygialis meridionalis (Pum) and two poison frog species that carry alkaloid toxins in the wild (Phyllobates terribilis, BTX, and Dendrobates tinctorius, histrionicotoxin HTX, and pumiliotoxin, PTX). Surprisingly, we did not find the DIVS6 $\mathrm{N} \rightarrow \mathrm{T}$ mutation in any of these species even though the poison frog Navs possessed all other mutations suggested to contribute to BTX resistance (24). Further, the Pitohui and poison frog Navs were BTX sensitive. Although the DIVS6 N $\rightarrow$ T mutation 
reduced Pum Nav BTX sensitivity, in line with its effect in rat Nav1.4 (25), this same mutation failed to confer BTX resistance to poison frog Navs. Additionally, in all cases, DIVS6 N $\rightarrow$ T greatly compromised channel function, underscoring the tradeoffs between toxin-resistant mutations and fitness cost (29). Most surprising, poison frogs having BTX-sensitive Navs proved resistant to BTX poisoning and resisted poisoning by another small molecule toxin, saxitoxin (STX), despite expressing STX-sensitive Navs. A distantly related poison frog that does not carry BTX (Mantella) also proved resistant to BTX and STX. Hence, our studies refute the idea that target $\mathrm{Na}_{V}$ mutations are singularly responsible for BTX autoresistance in poisonous birds and frogs, and suggest that other, more general mechanisms such as toxin sequestration $(13,14,30,31)$ defend against autointoxication. 


\section{Results}

\section{Cloning and characterization of skeletal and cardiac Pitohui Navs}

Pitohui is one of only a few bird genera known to carry $\operatorname{BTX}(3,4,32)$ and has BTX levels in its skeletal and cardiac muscles that should alter $\mathrm{Nav}_{v}$ function (8). To investigate possible mechanisms of BTX resistance, we used a Pitohui genomic DNA library to identify and assemble genes for two Pitohui uropygialis meridionalis Navs, skeletal muscle Pum Nav1.4 (Fig. S1) and cardiac Pum Nav1.5 (Fig. S2). Primary sequence alignment showed extensive similarities between Pum Nav1.4, Pum Nav1.5, and other vertebrate homologs ( 73\% amino acid identity) (Figs. S1 and S2), including hallmark Nav features, such as: a selectivity filter 'DEKA' motif, canonical RXXR repeats in S4 in all four voltage sensor domains, and the 'IFM' motif responsible for fast inactivation (33) (Figs. S1 and S2).

Whole-cell patch clamp electrophysiology of Pum Nav1.4 and Pum Nav1.5 transfected into HEK293 cells demonstrated that both have fast voltage-dependent activation followed by a fast and complete voltage-dependent inactivation typical of Navs (Figs. 1a-b, S3a-b, and Tables 1 and S1) similar to human Nav1.4, Hs Nav1.4, recorded under identical conditions (Fig. 1c-d, Tables 1 and S1). Because Navs can harbor resistance mutations to other small molecule toxins (13, 14, 17, 20), we anticipated that the Pitohui Navs might be BTX-resistant. Surprisingly, application of $10 \mu \mathrm{M}$ BTX drastically altered the function of both Pum Navs, yielding typical BTX-induced functional consequences: a hyperpolarized shift in the voltage-dependency of activation $\left(\Delta \mathrm{V}_{1 / 2 \mathrm{BTX}}=-33.6 \pm 1.2\right.$ and $-37.4 \pm 1.8$ for Pum Nav1.4 and Pum Nav1.5, respectively $)$, reduced inactivation, and enhanced tail currents $(10,11)$ (Figs. 1a-b and S3a-b, Table 1). The BTX-induced activation curve follows a double Boltzmann function in which the first and second components arise from BTX-bound and unmodified channels, respectively (34). Notably, the BTX-induced changes were equivalent to those elicited by BTX application to Hs Nav1.4 $\left(\Delta \mathrm{V}_{1 / 2 \mathrm{BTX}}=-35.9 \pm 1.8\right)$ (Fig. 1d, Table 1). These data demonstrate that despite the fact that Pitohui carry BTX in its skeletal muscles and heart (8), their skeletal and cardiac Navs are BTXsensitive. Thus, autoresistance cannot originate from altered BTX sensitivity in the two most likely target channels exposed to lethal BTX levels.

Navs are often co-expressed with auxiliary $\beta$ subunits that can alter their biophysical (35) and pharmacological $(36,37)$ properties. To test whether this subunit could impact BTX resistance, we identified the Pitohui uropygialis meridionalis gene encoding for a transmembrane protein bearing the key features of $\mathrm{Nav}_{v} \beta 2$, including a pair of conserved disulfides (38). (Fig. S3c). 
Co-expression of Pum Nav $\beta 2$ with Pum Nav1.4 had no impact on channel biophysical properties or on BTX responses relative to Pum Nav1.4 alone (Fig. S3d-e, Tables 1 and S1). Thus, Pum Nav1.4 alone and Pum Nav1.4 in combination with Pum Nav $\beta 2$ failed to showed evidence of BTX-resistant channels.

\section{Cloning and characterization of poison frog Navs}

Poison frogs in the genus Phyllobates (Family Dendrobatidae) are the most well-known BTX carriers $(2,27,39)$. A number of studies have identified amino acid substitutions hypothesized to contribute to poison frog $\mathrm{Nav}$ BTX resistance (24-26). However, cloning and functional characterization of poison frog Navs is noticeably absent from the literature. We used skeletal muscle from captive-raised members of two representative poison frog species, one that carries high levels of BTX in the wild, Phyllobates terribilis $(P t \operatorname{Nav1.4)}(23,24,40)$ and one not known to carry BTX, Dendrobates tinctorius (Dt Nav1.4)(24), to clone poison frog Nav1.4s. Consistent with evolutionary relationships between the two species $(24,26)$, Pt Nav1.4 and Dt Nav1.4 were highly similar to each other ( $\sim 95 \%$ amino acid identity) and other vertebrate Navs $(\sim 73 \%$ amino acid identity), and bore all Nav hallmarks (Fig. S1). Importantly, their DIS6 and DIVS6 sequences were identical to those reported previously (24) with the remarkable absence in Pt Nav1.4 of the proposed BTX-resistance mutation DIVS6 N $\rightarrow$ T (Pt Nav1.4 Asn1600, Pt Nav1.4 N1584T (rat numbering) (25)) (Fig. S1, Table S2). Genomic DNA sequencing covering the Pt Nav1.4 DIVS6 yielded nucleotide sequences identical to those obtained from cDNA and cross-validated the absence of the DIVS6 $\mathrm{N} \rightarrow \mathrm{T}$ substitution. These findings are consistent with the observation that the DIVS6 N1600T substitution has a very low frequency among wild $P$. terribilis (26). Besides the prior reported amino acid variants (24), Pt Nav1.4 and Dt Nav1.4 had 93 additional positions distributed throughout the channel that differed from conserved bird, human, and rat Nav1.4 residues (Fig. S4, Table S2). Although we could readily sequence the Pt Nav1.4 and Dt Nav1.4 genes, both proved prone to recombination and deletion upon passage through $E$. coli, rendering the native DNA sequences impossible to handle. To solve this problem, we re-designed the codon usage to preserve the amino acid sequence of both. These re-designed genes were well behaved and allowed us to conduct electrophysiological characterization of Pt Nav1.4 and Dt Nav1.4 in mammalian and amphibian expression systems.

Whole-cell patch clamp electrophysiology of HEK293 cells transfected with Pt Nav1.4 and $D t$ Nav1.4 yielded voltage-dependent channels that matched the properties of both Pum and $H s$ Nav1.4s (Fig. 1e-h, Tables 1 and S1). Strikingly, both poison frog Navs had the same response as Pum and $H s$ Nav1.4 to $10 \mu \mathrm{M}$ BTX (Fig. 1e-h), $\left(\Delta \mathrm{V}_{1 / 2 \mathrm{BTX}}=-30.0 \pm 2.1\right.$ and $-37.9 \pm 2.0 \mathrm{mV}$ for 
Pt Nav1.4 and Dt Nav1.4, respectively), demonstrating that these poison frog channels are not resistant to $\mathrm{BTX}$ and ruling out the possibility that the $>90$ amino acid variants between poison frog and human channels, including the previously proposed changes in DIS6 and DIVS6 (24), could cause BTX-resistance.

Because expression of amphibian channels in an amphibian cell could provide a more native-like context, we also expressed Pt Nav1.4 and Dt Nav1.4 in Xenopus oocytes and examined their function by two-electrode voltage clamp. Both channels had biophysical parameters that matched those measured in mammalian cells (Fig. S5a-d, Table 1). Further, BTX application caused the strong hallmark functional modification observed for all of the other channels we studied (Figs 1 , S3a-b and d-e, and S5a-d), including voltage-dependent activation shifts comparable to those measured in mammalian cells $\left(\Delta \mathrm{V}_{1 / 2} \mathrm{BTX}=-33.1 \pm 3.2\right.$ and $-30.0 \pm 2.1$; and $-37.8 \pm 6.1$ and $-37.9 \pm 2.0 \mathrm{mV}$ for Pt Nav1.4 and Dt Nav1.4 expressed in Xenopus oocytes and HEK293 cells, respectively). The shift was more complete in oocytes (cf. Figs. $1 \mathrm{f}$ and $\mathrm{h}$ and S5b and d), a result that likely originates from the fact that for technical reasons BTX was injected into the oocytes rather than applied by bath application as for mammalian cells. Taken together, the biophysical characterization of Pt Nav1.4 and Dt Nav1.4 demonstrates that these channels are not BTX-resistant. Our combined observations that Navs from two classes of BTX-carrying animals, Pitohui and $P$. terribilis, are vulnerable to BTX challenge the idea that Nav mutation is the BTX autoresistance strategy as suggested for poison frogs such as $P$. terribilis $(24,25)$.

\section{DIVS6 $\mathrm{N} \rightarrow \mathrm{T}$ mutation fails to confer BTX resistance to poison frog Navs}

Because the DIVS6 $\mathrm{N} \rightarrow$ T mutation was absent from Navs of BTX-bearing species, we wondered whether the observation that DIVS6 $\mathrm{N} \rightarrow \mathrm{T}$ could confer BTX resistance to rat Nav1.4 (25) was impacted by the $>90$ amino acid differences between poison frog and mammalian Navs (Fig. S4, Table S2). Therefore, we placed the DIVS6 $\mathrm{N} \rightarrow \mathrm{T}$ mutation in poison bird, human, and poison frog Nav1.4s (Pum Nav1.4 N1609T, Hs Nav1.4 N1591T, Pt Nav1.4 N1600T, and Dt Nav1.4 N6100T) and measured its effects on channel function and BTX sensitivity. Consistent with studies of rat Nav1.4 DIVS6 $\mathrm{N} \rightarrow \mathrm{T}$ (25), DIVS6 $\mathrm{N} \rightarrow \mathrm{T}$ eliminated the ability of BTX to block inactivation and induce large tail currents in Pum Nav1.4 and Hs Nav1.4 (Fig. 2a-d). Nevertheless, the bird and human Nav1.4s were not rendered completely BTX resistant. Application of $10 \mu \mathrm{M}$ BTX shifted the voltage-dependent activation of both channels, making them more easily opened by voltage $\left(\Delta \mathrm{V}_{1 / 2 \mathrm{BTX}}=-11.4 \pm 3.2\right.$ and $-14.2 \pm 5.5 \mathrm{mV}$ for Pum Nav1.4 N1609T and Hs Nav1.4 N1591T, respectively) (Fig. 2b and d, Table 1). Further, the BTX-induced double Boltzmann was lost (Fig. 2b and d), suggesting an enhanced BTX affinity. Due to its limited effectiveness in blocking 
the effects of BTX in the bird and human channels, we revisited the consequences of the DIVS6 $\mathrm{N} \rightarrow \mathrm{T}$ mutation in rat Nav1.4 (Rn Nav1.4). Similar to the results with bird and human channels, DIVS6 $\mathrm{N} \rightarrow \mathrm{T}$ reduced but did not eliminate $\mathrm{Rn}$ Nav1.4 channel BTX sensitivity (Figure S6a-d). Application of $10 \mu \mathrm{M}$ BTX shifted the voltage-dependent activation of both $R n \mathrm{Nav} 1.4$ and $R n \mathrm{Na}_{\mathrm{V}} 1.4 \mathrm{~N} 1584 \mathrm{~T}$ making them more easily opened by voltage $\left(\Delta \mathrm{V}_{1 / 2 \mathrm{BTX}}=-45.6 \pm 0.4\right.$ and -40.3 $\pm 2.8 \mathrm{mV}$ for $R n$ Nav1.4 and $R n$ Nav1.4 N1584T, respectively) (Fig S6b and d, Table 1). Thus, DIVS6 $\mathrm{N} \rightarrow \mathrm{T}$ was unable to mitigate the effects of BTX completely in any Nav orthologues.

In all three contexts, DIVS6 $\mathrm{N} \rightarrow \mathrm{T}$ also affected channel biophysical properties (Figs. S6e-g, S7a-h, Tables 1, S1 and S3). DIVS6 N $\rightarrow$ T rendered Pum Nav1.4, Hs Nav1.4 and Rn Nav1.4 more difficult to open, shifting the activation voltage dependence to depolarizing potentials $\left(\Delta \mathrm{V}_{1 / 2}=+20.5 \pm 1.5,+16.9 \pm 2.7\right.$, and $+18.0 \pm 0.6 \mathrm{mV}$ for Pum Nav1.4 N1609T, Hs Nav1.4 $\mathrm{N} 1591 \mathrm{~T}$, and Rn Nav1.4 N1584T, respectively) (Figs. S6e, S7b and f, Tables 1 and S3), and made the channels easier to inactivate, shifting the voltage dependence of steady state inactivation towards hyperpolarizing potentials $\left(\Delta \mathrm{V}_{1 / 2}\right.$ inact $=-10.3 \pm 2.1, \quad-9.8 \pm 1.2$ and $-27.0 \pm 0.4 \mathrm{mV}$ for Pum Nav1.4 N1609T, Hs Nav1.4 N1591T and Rn Nav1.4 N1584T, respectively) (Figs. S6f, S7c and g, Tables S1 and S3). Further, DIVS6 $\mathrm{N} \rightarrow \mathrm{T}$ diminished Pum Nav1.4 N1609T, Hs Nav1.4 N1591T and Rn Nav1.4 N1584T current densities by 79\%, 55\% and 90\%, respectively (Figs. S6a, c, and g, and S7a, d, e, and h, Tables 1 and S3). Thus, the DIVS6 $\mathrm{N} \rightarrow \mathrm{T}$ change incurs a substantial functional cost.

To probe the DIVS6 Asn site further, we examined the consequences of mutation to alanine in Pum Nav1.4. Pum Nav1.4 N1609A phenocopied the biophysical changes measured for N1609T producing channels that were more difficult to open $\left(\Delta \mathrm{V}_{1 / 2}=+19.6 \pm 1.6\right)$, easier to inactivate $\left(\Delta \mathrm{V}_{1 / 2 \text { inact }}=-13.4 \pm 1.6\right)$, and had current density reduced by $76 \%$ (Fig. S7a-d, Tables 1 , S1, and S3), in agreement with the reduced channel activity reported for the corresponding rat Nav1.4 mutant $(41,42)$. These biophysical changes match those of the BTX-resistant Pum Nav1.4 N1609T; however, Pum Nav1.4 N1609A retained all of the classical BTX responses such as reduction of inactivation, enhanced tail current, and a leftward shift of the activation voltage dependence (Fig. S7i-j, Tables 1 and S3). The failure of the N1609A to diminish BTX sensitivity shows that the reduction of BTX sensitivity in Pum Nav1.4 N1609T, Hs Nav1.4 N1591T, and $R n$ Nav1.4 N1584T is a specific effect of the threonine mutation, and not a consequence of the changes in channel biophysical properties or current density reduction (Table S3).

To our surprise, placing DIVS6 $\mathrm{N} \rightarrow \mathrm{T}$ in both poison frog Nav1.4s failed to blunt the effects of BTX on channel activation and inactivation (Fig. 2e-h) $\left(\Delta \mathrm{V}_{1 / 2 \mathrm{BTX}}=-30.0 \pm 2.1,-30.8 \pm 2.5 \mathrm{mV}\right.$ for 
Pt Nav1.4 and Pt Nav1.4 N1600T, respectively and $-37.9 \pm 2.0$ and $-37.8 \pm 1.8$ for Dt Nav1.4 and Dt Nav1.4 N1600T, respectively). Hence, even though the DIVS6 $\mathrm{N} \rightarrow \mathrm{T}$ change reduces Pum Nav1.4, Hs Nav1.4, and Rn Nav1.4 BTX responses (Figs. 2a-d, S6a-d), this same change fails to affect the BTX sensitivity of poison frog Navs. Unlike its effects in Pum, Hs, and Rn Navs, DIVS6 $\mathrm{N} \rightarrow \mathrm{T}$ did not cause major changes in poison frog Nav biophysical properties, shifting only the inactivation voltage dependence by $-10 \mathrm{mV}$ while leaving the activation voltage dependence and current density unchanged (Fig. S8a-C, Tables 1, S1, and S3). However, expression of Pt Nav1.4 N1600T and Dt Nav1.4 N1600T in Xenopus oocytes revealed dramatic reductions in channel activity (Fig. S8d-h) that prevented measurement of channel biophysical properties and BTX responses. These results reveal that DIVS6 $\mathrm{N} \rightarrow \mathrm{T}$ is detrimental to function and may interfere with channel folding and maturation in a manner that is accentuated at lower temperatures, such as those used to store incubate the oocytes. This context-dependent loss of function indicates that the DIVS6 $\mathrm{N} \rightarrow \mathrm{T}$ variant exacts a functional cost that, together with its ineffectiveness in endowing poison frog Navs with BTX resistance, refutes the idea that DIVS6 $N \rightarrow T$ could serve as an effective BTX autoresistance mechanism.

\section{Cost of the conserved $\mathrm{N} \rightarrow \mathrm{T}$ mutation is context dependent}

The varied outcomes of DIVS6 $\mathrm{N} \rightarrow$ T on BTX sensitivity among the poison bird, human, rat, and poison frog Navs highlight the importance of context in determining the functional consequences of mutations. Because the equivalent residue is conserved in all four S6 helices (Figs. S1 and S2), we systematically introduced S6 $\mathrm{N} \rightarrow \mathrm{T}$ into each of the Pum Nav1.4 S6 segments and measured channel properties and BTX responses to investigate the question of context dependent effects further (Fig. 3). Whole cell patch clamp recordings from HEK293 cells transfected with these mutant channels revealed clear, domain-specific differences. Contrasting the effect of DIVS6 $\mathrm{N} \rightarrow \mathrm{T}$ (Fig. S7b), voltage-dependent activation of channels having the $\mathrm{N} \rightarrow \mathrm{T}$ mutation in DI, DII, or DIII (Pum Nav1.4 N432T (DI), Pum Nav1.4 N830T (DII), and Pum Nav1.4 $\mathrm{N} 1306 \mathrm{~T}$ (DIII)) was unchanged relative to wild-type $\left(\mathrm{V}_{1 / 2}=-20.2 \pm 1.9,-22.4 \pm 1.0,-26.7 \pm 1.0\right.$, and -23.4 $1.0 \mathrm{mV}$ for Pum Nav1.4 N432T (DI), Pum Nav1.4 N830T (DII), Pum Nav1.4 N1306T (DIII), and Pum Nav1.4, respectively) (Figs. 3a-j, S9a-b, and Tables 1 and S3). By contrast, we found varied effects on steady state inactivation. DI and DIII changes showed wild-type like behavior, whereas the DIl mutant had a $\sim 10 \mathrm{mV}$ hyperpolarizing shift $\left(\mathrm{V}_{1 / 2}\right.$ inact $=-61.2 \pm 1.6,-75.0 \pm 0.9,-65.3 \pm 1.0$, and $-64.2 \pm 1.3 \mathrm{mV}$ for Pum Nav1.4 N432T (DI), Pum Nav1.4 N830T (DII), Pum Nav1.4 N1306T (DIII), and Pum Nav1.4, respectively) (Fig. S9c, Tables S1 and S3). All three had strong BTX responses similar to wild-type 
$\left(\Delta \mathrm{V}_{1 / 2 \mathrm{BTX}}=-30.8 \pm 2.1,-31.6 \pm 2.0,-36.9 \pm 1.4\right.$, and $-33.6 \pm 1.2 \mathrm{mV}$ for Pum Nav1.4 N432T (DI), Pum Nav1.4 N830T (DII), Pum Nav1.4 N1306T (DIII), and Pum Nav1.4, respectively) (Fig. 3, Tables 1 and S3). Thus, the only site where the conserved S6 $\mathrm{N} \rightarrow \mathrm{T}$ change affects BTX responses is in DIVS6, in line with its proposed contribution to the BTX binding site (25).

As with the biophysical changes, the effects on current density from placing the $\mathrm{N} \rightarrow \mathrm{T}$ change in different channel domains were not uniform. The DIS6 and DIIIS6 $\mathrm{N} \rightarrow$ T mutants had current densities matching wild-type (Figs. 3a, c, g, S9a and d, and Tables 1 and S3), whereas, DIIS6 $\mathrm{N} \rightarrow \mathrm{T}$ lowered the current density and was more detrimental to channel activity than DIVS6 N1609T or N1609A (Figs. 3a, e, S9a and d, and Tables 1 and S3). Together, these data show that there is no correlation between changes in channel biophysical properties and the acquisition of BTX resistance and are in line with the results from DIVS6 $\mathrm{N} \rightarrow \mathrm{T}$ and $\mathrm{N} \rightarrow \mathrm{A}$ mutants (Figs. 2, S7, and S8, Table S3).

Consideration of the conserved S6 asparagine structural context provides insight into the context-dependent effects. The two $\mathrm{S} 6$ sites where $\mathrm{N} \rightarrow \mathrm{T}$ has no impact on channel biophysics, BTX responses, or current density, DIS6 and DIIIS6, occupy positions that are partially exposed to the channel inner pore (Fig. S9e-f). By contrast, the two positions that affect channel biophysics and current density, DIIS6 and DIVS6, interact with the S4-S5 linkers (43) (Fig. S9e-f) and altering these buried sites comes with substantial functional costs. Hence, DIVS6 $\mathrm{N} \rightarrow \mathrm{T}$ carries major disadvantages for protecting animals such as Pitohui and poison frogs against BTX autointoxication.

\section{Toxin-free poison frogs are BTX resistant in the absence of the DIVS6 $\mathrm{N} \rightarrow \mathrm{T}$ mutation}

The surprising observation that Navs from BTX-carrying birds and frogs remain BTX-sensitive raised the question of whether the species from which we cloned the channels were actually BTX resistant. Because of difficulties in obtaining live animals, we were unable to investigate Pitohui BTX resistance. Captive-raised poison frogs lack BTX, as this toxin is acquired in the wild from their diet $(5,6)$. Thus, it was possible that the toxin-free poison frogs used to clone Navs were not BTX resistant due to the absence of selective pressure from the toxin, a possibility underscored by the high functional cost of DIVS6 $\mathrm{N} \rightarrow \mathrm{T}$ (Figs. 2e-h and S8, Tables 1, S1 and S3). To test whether captive-raised poison frogs were BTX resistant, we conducted a series of toxin challenge experiments using five different frog species: two non-poisonous frogs (Xenopus laevis and Polypedates leucomystax), two captive-raised poison frogs that carry alkaloid toxins in the wild (Phyllobates terribilis, BTX; Dendrobates tinctorius, histrionicotoxins HTX, and pumiliotoxins, PTX), and an unrelated captive-raised Malagasy poison frog that carries PTX rather than BTX in 
the wild (Mantella aurantiaca) and represents an independent evolutionary origin of chemical defenses (44-46). We challenged these animals with three different toxins that target Navs: BTX, and two guanidinium toxins that act by a pore-blocking mechanism, saxitoxin (STX) $(47,48)$, and tetrodotoxin (TTX) (48).

We assessed the duration of recovery from anesthesia-induced paralysis following intramuscular injection of each toxin at 20 times the lethal dose based on values for mice $\left(L_{50}\right)$ by monitoring how long it took the frog to show clear motor activity relative to injection of a phospho-buffered saline (PBS) control. Following BTX injection, Xenopus laevis and Polypedates leucomystax displayed an accelerated recovery from anesthesia that was at least two times faster than PBS injections (PBS and BTX recovery times: $29 \pm 1 \mathrm{~min}$. and $15 \pm 5 \mathrm{~min}$., and $169 \pm 12 \mathrm{~min}$. and $70 \pm 20$ min. for $X$. laevis and $P$. leucomystax, respectively) (Fig. 4a-b and f, Table S4). After the initial recovery, BTX was ultimately lethal to $X$. laevis (Fig.4a and f). By contrast, BTX injection did not change the anesthesia recovery time or kill any of the poison frogs, regardless of whether they carry BTX in the wild environment ( $P$. terribilis) or are naturally BTX-free but harbor other alkaloid toxins (D. tinctorius, M. aurantiaca) (Fig. 4c-f, Table S4). Reponses to STX also revealed differences between non-poisonous and poison frogs. STX injection was lethal to $X$. laevis and $P$. leucomystax (Fig. 4a-b and f), whereas all three poison frogs fully recovered from anesthesia after STX injections (Fig. 4c-f). TTX was lethal to $X$. laevis (Fig. 4a and f). Although TTX caused extended paralysis in all other tested frogs, it was not lethal (Fig. 4a-f, Table S4). Thus, all tested poisonous frogs showed resistance to all three toxins while non-poisonous frogs were vulnerable to either BTX and STX (P. leucomystax) or all three toxins (X. laevis) (Fig. 4f).

The striking differences in BTX-induced accelerated recovery from anesthesia between the non-poisonous and poisonous species was unexpected. Studies of eukaryotic and prokaryotic Navs suggest that BTX and local anesthetics, such as the tricaine used for frog anesthesia, have overlapping binding sites within the channel pore (49-52). We considered that the differences in BTX-dependent accelerated recovery from anesthesia were a physiological manifestation of this molecular competition and indicated that BTX was engaging the target channels in non-poisonous frogs but not in poison frogs. Hence, we tested whether tricaine and BTX produced competing effects on Pt Nav1.4 and Dt Nav1. 4. Consistent with its anesthetic effects on the frogs, $0.5 \mathrm{mM}$ tricaine inhibited both poison frog Navs and had similar effects on the Pum Nav1.4 control (Fig. S10a-c and g). Subsequent BTX injection into the same tricaine-treated oocytes caused a complete relief of the tricaine block (Fig. S10a-C and g), in line with a direct competition between tricaine and BTX. By contrast, in the absence of tricaine, this dramatic BTX-induced increase in 
peak current was absent (Fig. S10d-f and h). These data demonstrate that the poison frog Navs are competent for BTX-tricaine competition. Hence, the differences in BTX-induced accelerated recovery from anesthesia reflect the direct competition of the two compounds on the channel in the non-poisonous frogs and suggest that the poison frogs have a means to prevent BTX form engaging their Navs.

The poison frog STX resistance we observed could be explained by a lack of Nav sensitivity to this toxin. To test this possibility, we compared the responses of Pt Nav1.4, Dt $\mathrm{Na}_{\vee} 1.4$, and Pum Nav1.4 as a control, to STX and TTX as the former had minimal effect on the poison frogs, whereas the latter caused potent paralysis (Fig. 4, Table S4). Extracellular application of increasing STX concentrations inhibited all three Navs with a nanomolar response that matched other Navs $(47,53,54)\left(\mathrm{IC}_{50}=12.6 \pm 1.4 \mathrm{nM}, 14.6 \pm 0.6 \mathrm{nM}\right.$ and $7.3 \pm 0.5 \mathrm{nM}$ for Pt Nav1.4, Dt Nav1.4 and Pum Nav1.4, respectively) (Fig. S11a-d). Pt Nav1.4 and Dt Nav1.4, and the control Pum Nav1.4 also had nanomolar TTX responses, similar to $H s$ Nav1.4 (55), (IC $50=21.3 \pm 1.0 \mathrm{nM}$, $40.8 \pm 1.8 \mathrm{nM}$, and $6.2 \pm 0.4 \mathrm{nM}$ for Pt Nav1.4, Dt Nav1.4, and Pum Nav1.4, respectively) (Fig. S11e-h). Thus, the ability of the poison frogs to resist STX does not arise from their Navs having some unusual resistance to the toxin (Figs. 4, S11d and Table S4). The resistance of poison frogs to the effects of BTX and STX contrasts with the effects of TTX and is not consistent with the high sensitivity of their Nav1.4s to all three toxins. These findings suggest that mechanisms, such as toxin sequestration, may prevent BTX and STX from reaching their target Navs. 


\section{Discussion}

Poisonous organisms that use toxins as defensive molecules must avoid autotoxication. Such resistance has been proposed to arise from three strategies: 1) acquisition of target protein toxin resistance mutations, 2) toxin sequestration, and 3) enhanced detoxification or elimination capacity $(13,14)$. Support for the first mechanism includes prominent examples of TTX-resistant Navs in toxin-bearing species and their predators (18-22), STX-resistant Navs in mollusks (17), and epibatidine-resistant $\mathrm{nAChRs}$ in poison frogs (16). Because of these examples and the suggestion that the Nav DIVS6 $\mathrm{N} \rightarrow$ T mutation might confer BTX-resistance to poison frogs (25), we expected to find toxin resistant mutants in poison bird and frog Navs. Instead, we found multiple lines of evidence demonstrating that Navs from both poison birds and frogs are highly sensitive to BTX and lack the DIVS6 $\mathrm{N} \rightarrow \mathrm{T}$ change. Further, even though the DIVS6 $\mathrm{N} \rightarrow \mathrm{T}$ mutation alters the BTX-responses of bird, human, and rat Nav1.4s (Figs. 2a-d and S6a-d), it failed to have any effect on the BTX-sensitivity of poison frog Navs (Fig. 2e-h), a result that highlights the importance of vetting putative toxin-resistance mutations in the context of the native channel (16).

How amino acid changes compensate for mutations that alter function is complex and can arise from effects at positions that are far apart in the protein structure (56-59). There are >90 amino acid differences between the poison frog and human Nav1.4s (Fig. S4, Table S2) and it is not obvious which variants in the frog Navs suppress the ability of DIVS6 $N \rightarrow T$ to affect BTX responses. The importance of context is further evident from the fact that even though the Asn position is conserved in all four $\mathrm{Nav}$ pore domain subunits, the functional consequences of the $\mathrm{N} \rightarrow \mathrm{T}$ change are domain-dependent (Fig. 3, Table S3). These factors, together with the absence of DIVS6 $\mathrm{N} \rightarrow \mathrm{T}$ in BTX-bearing birds and frogs (Fig. S1-S2) $(24,26)$ and its ineffectiveness in poison frog Navs rule out the target-alteration hypothesis for BTX resistance.

Endowing a protein with a new function through mutation often incurs a cost, particularly with respect to protein stability (57-59). Our data show that the DIVS6 $\mathrm{N} \rightarrow \mathrm{T}$ change in bird, human, rat, and frog Navs carries substantial functional costs that affect every aspect of channel function, in line with its role in coupling the pore to the voltage-sensor domain in Navs (42), by inducing changes that render the channels more difficult to open, more readily inactivated, and that reduce current density (Table S3), an effect that likely reflects stability penalties that impact channel biogenesis. Similar perturbations of $\mathrm{Na} v$ inactivation and reduction of current levels have profound physiological consequences (60) and are linked to a variety of channelopathies (61), underscoring the organism-level fitness problems incurred by changes in $\mathrm{Na}_{v}$ biophysical properties. These 
substantial fitness costs, as well as the inability of the DIVS6 $\mathrm{N} \rightarrow \mathrm{T}$ mutation to affect the BTX responses of poison frog Navs are consistent with the low frequency of this variant in wild $P$. terribilis (26) and its absence from the BTX-bearing $P$. aurotaenia poison frog (24). Other studies of ion channel toxin resistance mutants have uncovered various degrees of functional costs that may be compensated by amino acid changes at additional sites in the channel $(16,62)$. Hence, the effectiveness of developing a toxin-resistant channel via mutation is highly dependent on the cost for evolving this new function and the extent to which functional costs can be mitigated by additional changes.

Poison frogs lacking the Nav1.4 DIVS6 $\mathrm{N} \rightarrow \mathrm{T}$ change withstand BTX levels that affect non-poisonous frogs (Fig. 4, Table S4), in line with previous studies (23). In non-poisonous frogs, we find clear in vivo physiological antagonism between the channel blocker, tricaine, and the channel opener, BTX, that indicates both compounds access the target Navs. This antagonism is absent in poison frogs (Fig. 4, Table S4) even though it can occur at the molecular level of the channel (Fig. S10). Further, the resistance of poison frogs to BTX and STX contrasts with the effects of TTX and is not consistent with the high sensitivity of their Nav1.4s to all three toxins (Figs. 4, S10-S11). Together these observations suggest that poison frogs have a means to prevent BTX and STX from reaching their Navs. It is notable that other frogs resist STX poisoning $(31,63,64)$ and it is thought that the soluble STX binding protein saxiphilin $(13,31,65)$ acts as a 'toxin sponge' to sequester and neutralize the lethal effects of this and possibly other neurotoxins $(13,14,30,31,66,67)$.

If BTX-bearing animals do not use BTX-resistant Navs to avoid autointoxication, how do they survive? Apart from the absence of BTX-resistant Navs, the diversity among $>800$ poison frog alkaloid toxins (44), the seasonal and geographical variation of these toxins, and ability to affect multiple ion channels (2) pose major challenges for evolving toxin resistant channels. Enhanced detoxification via metabolic toxin destruction would also not be useful as these poisonous organisms need to handle and store the toxins to deploy them against predators. By contrast, sequestration strategies not only offer a general means of toxin protection, but could act in pathways involved in safely transporting and concentrating toxins in key defensive organs such as the skin (32). The fact that toxin-based chemical defense systems have evolved independently four times in neotropical poison frogs (Dendrobatids)(2), in Malagasy poison frogs (46), and in multiple lineages of poisonous birds (including Pitohui and Ifrita) $(3,4)$ supports the idea that such general sequestration mechanisms may underlie toxin autoresistance. Although no BTX-binding proteins have been yet identified, high-affinity toxin binding proteins for STX in frogs, saxiphilin 
$(30,31,65)$ and TTX in pufferfish, Pufferfish Saxitoxin and Tetrodotoxin Binding Protein $(68,69)$ are known and have been proposed to prevent autointoxication through sequestration (13). Characterizing how such toxin-binding proteins protect hosts from autointoxication, alone or together with specialized toxin transport pathways, should provide new insights into the fundamental mechanisms of toxin autoresistance, expand understanding of how organisms handle a range of chemical insults, and may lead to the discovery of antidotes against various toxic agents. 


\section{Acknowledgements}

We thank J. McGlothlin (Virginia Tech) for providing annotated sequences of crow Navs, G. Loussouarn (l'institut du thorax, Nantes) for providing human Nav clones, Z. Chen for technical assistance, and K. Brejc for comments on the manuscript. We thank the Papua New Guinea National Research Institute and Department of Environment and Conservation for permission to conduct fieldwork, the generous people of Bonua Village for assistance in the field permission to work on their land, and the National Geographic Society for funding the fieldwork. This work was supported by grants NIH-NHLBI R01-HL080050 and NIH-NIDCD R01 DC007664 to D.L.M., NSF-1822025 to L.A.O, partial support of this work from NIH-NIGMS GM117263-01A1 to J.D.B, an American Ornithological Society Wetmore Research Award to M.E.K., an NIH-NIGMS F32GM116402 postdoctoral fellowship to R.A.C., and an American Heart Association postdoctoral fellowship to F.A.-A.

\section{Author Contributions}

F.A.-A., M.E.K., R.A.C., C.E.G., J.D.B, J.P.D. and D.L.M. conceived the study and designed the experiments. F.A.-A., N.D.R., R.A.C., and C.E.G. performed patch clamp electrophysiology experiments and analyzed the data. F. A.-A. performed de novo Nav cloning, molecular biology experiments, two-electrode voltage clamp electrophysiology experiments, toxin challenge experiments, and analyzed the data. M.E.K. and J.P.D. constructed and analyzed Pitohui genomes. J.D.B. synthesized BTX. J.D.B., J.P.D, and D.L.M analyzed data and provided guidance and support. F.A.-A., L.A.O., J.D.B., J.P.D, and D.L.M. wrote the paper.

\section{Competing interests}

J.D.B. is a cofounder and holds equity shares in SiteOne Therapeutics, Inc., a start-up company interested in developing subtype-selective modulators of sodium channels.

The other authors declare no competing interests.

\section{Data and materials availability}

Sequences of Pum Nav1.4, Pum Nav1.5, Pum Navß2, Pt Nav1.4, and Dt Nav1.4 will be deposited with $\mathrm{NCBI}$ and released upon publication.

Requests for material to D.L.M. 


\section{Materials and Methods}

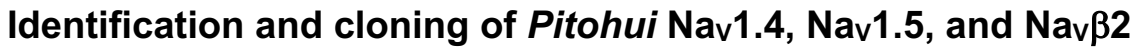

Genomic DNA from Pitohui uropygialis meridionalis (Family Oriolidae) blood and tissue was extracted using DNeasy kits (Qiagen) to create whole genome sequence libraries for the poisonous Pitohui birds. Tissue samples were collected in 1989 from near the village of Bonua, Central Province, Papua New Guinea (1008'S by $\left.149^{\circ} 10^{\prime} 30^{\prime \prime} \mathrm{E}\right)$, stored in ethanol in the field, and frozen since being in the lab. Two genome sequence libraries were created using Illumina Nextera kits. One library had a target insert size of 500-640, and occupied a full run on the MiSeq genetic analyzer using 300-base paired-end reads, and the second library had a target insert size of 640-709 and occupied a full lane of a HiSeq 2500 in rapid run mode using 150-base pairedend reads.

The MiSeq run returned 16,279,946 paired-end reads. The program BBmerge version 4.0 (DOE: Joint Genome Institute; https://jgi.doe.gov/data-and-tools/bbtools/bb-tools-user-guide/bbmergeguide/) was used to join the forward and reverse reads into a single long read. $7,050,488$ of the read pairs $(\sim 43 \%)$ were joined, and the remaining reads were retained as paired-end reads or single reads for later analyses. The average size of merged reads was 542.6 bases. All reads were then trimmed using Trimmomatic (70) for minimum length, removing adapters, and performing basic quality filtering. All unmerged and unpaired reads were combined into a single fastq file.

The HiSeq run returned 150,979,291 paired-end reads. We removed adapters, trimmed for minimum length, and performed basic quality filtering using Trimmomatic (70). 130,607,604 pairs of reads $(86.51 \%)$ passed filtering, plus another 9,199,536 (6.09\%) of forward only reads passed filter, and 3,342,519 (2.21\%) of reverse reads only passed filter. These two read sets (the MiSeq and the HiSeq Illumina datasets) composed the data for gene assembly.

Crows, Corvus brachyrhynchus and Corvus cornix, (Family Corvidae, from Joel McGlothlin, Virginia Tech) were the closest living relatives of Pitohuis that had a fully-annotated genome in the sodium channel gene regions. Crow sequences were used as reference sequences for BLAST searches of Pitohui sequences and for assisting with Pitohui Nav assembly. These sequences included the complete nuclear DNA sequence, with all exons, introns, and upstream and downstream UTRs.

To assemble the SCN4A gene from our Pitohui reads, we used the following pipeline, written as a bash shell script: First, we used BLATq version 1.0 .2 (https://github.com/calacademy- 
research/BLATq) which uses BLAT (Kent WJ. 2002. BLAT-The BLAST-Like Alignment Tool. Genome Research 12:656-664. DOI: 10.1101/gr.229202. Kent WJ. 2012. BLAT-The BLAST-Like Alignment Tool. Version 35. Available at https://users.soe.ucsc.edu/ kent.) to search both Illumina read sets for any sequences that aligned with the full genome sequences of one of the Corvus Nav1.4 sodium channel genes. We then used the script excerptBylDs v. 1.0.2 (https://github.com/calacademy-research/excerptBylDs) to create a new fastq files consisting of only the Illumina reads with strong BLATq scores. These files were combined into a single set of all BLAT "hits" using the Unix "cat" command. We then used the assembler SPAdes 3.9.0 (71) to perform an initial de novo assembly of reads in the hit file. We improved upon this assembly by using the genome assembly program PRICE ver. 1.2 (72), which iteratively extends the assembly using the assembled contigs from SPAdes as well as all of the existing Illumina read data. See the supplementary materials on the assembly bash shell script and options and parameters.

The assembled contigs from PRICE were loaded into Geneious (ver. 8.0 through 11.0.2). Within Geneious, we used BLAST to identify which contigs contained the Pitohui sequences. We created a BLAST database consisting of all of the assembled PRICE contigs, and we used each of the Corvus Nav1.4 exons to query the BLAST database of contigs using MegaBLAST. The top hits for each exon suggested which assembled contigs contained the Nav1.4 sequence, and typically several to all of the exons were found on the same contig. Assuming that the exon splice patterns were identical in crow and Pitohui, we aligned each of the crow Nav1.4 exon sequences to the top-hit Pitohui contig, and annotated the matching exon regions as the Pitohui SCN4A exons.

This assembly and annotation pipelines were repeated for primary voltage-gated sodium channels $\mathrm{Na}_{v} 1.5$ alpha subunit (SCN5A) as well as the $\mathrm{Nav} \beta$ subunit (SCN2B).

\section{Cloning of poison frog Navs}

Skeletal muscle was harvested from captive Phyllobates terribilis and Dendrobates tinctorius (Josh's frogs, Owosso, MI, USA) following euthanasia in accordance with UCSF IACUC protocol AN136799. Total RNA and total DNA were extracted using TRIzol ${ }^{\mathrm{TM}}$ Reagent (Thermo Fisher Scientific). Total RNA was reverse transcribed into cDNA using SuperScript ${ }^{\text {TM }}$ III First-Strand Synthesis System (Thermo Fisher Scientific). 5' and 3' end sequences of genes encoding for $P$. terribilis and $D$. tinctorius Nav1.4 were determined by DNA gel extraction and sequencing after rapid amplification of cDNA ends (RACE) using the SMARTer® RACE 5'/3' Kit (Takara Bio, USA) and internal primers designed from $P$. terribilis and D. tinctorius Nav1.4 S6 segment sequences (24). From these 5' end and 3' end sequences, new primers were designed from both 5' and 3' untranslated regions (UTR) of each gene, and were used to amplify full-length $P$. terribilis and $D$. 
tinctorius Nav1.4 genes by polymerase chain reaction (PCR) using Phusion $®$ HF (New England Biolabs). PCR products were gel extracted and sequenced to determine the full-length $P$. terribilis and $D$. tinctorius Nav1.4 gene sequences. Direct cloning of the full length PCR products of $P$. terribilis and $D$. tinctorius Nav1.4 channel genes into pCDNA3.1 proved problematic resulting unstable constructs prone to deletion. The codon optimized genes were synthesized for expression in human epithelium kidney cells (HEK293) (GenScript, Piscataway, NJ, USA), but were also found to prone to recombination upon insertion into pCDNA3.1. Finally, the gene sequences were redesigned to differ as much as possible from the original genes, synthesized (GenScript, Piscataway, NJ, USA), and cloned into pCDNA3.1. This strategy yielded stable constructs.

\section{Molecular biology}

For electrophysiology experiments, Hs Nav1.4 (GenBank: NM_000334.4), human Nav $\beta 1$ (GenBank: NM_001037.5), Pum Nav1.4, Pum Navß2, Pum Nav1.5, Pt Nav1.4, and Dt Nav1.4 were subcloned into pCDNA3.1 and Rn Nav1.4 (GenBank: Y17153.1) was subcloned into pZem228. All mutants were made using the QuikChange Site-Directed Mutagenesis Kit (Agilent) and validated by complete sequencing of the genes encoding for the proteins of interest.

\section{Patch clamp electrophysiology}

Human embryonic kidney cells (HEK 293) were grown at $37{ }^{\circ} \mathrm{C}$ under $5 \% \mathrm{CO}_{2}$, in a Dulbecco's modified Eagle's medium supplemented with $10 \%$ fetal bovine serum, 10\% L-glutamine, and antibiotics (100 IU ml- ${ }^{-1}$ penicillin and $100 \mathrm{mg} \mathrm{ml}^{-1}$ streptomycin). HEK293 cells were transfected (in 35-mm- diameter wells) using Lipofectamine 2000 (Invitrogen, Carlsbad, CA, USA) and plated onto coverslips coated with Matrigel (BD Biosciences, San Diego, CA, USA). Human and pitohui Navs were co-expressed with enhanced green fluorescent protein (EGFP), and human Nav $\beta 1$ or Pitohui Nav $\beta 2$. Poison frog Navs were co-expressed with EGFP. Transfected cells were identified visually by EGFP expression. A total of $2 \mu \mathrm{g}$ plasmid DNA ( $20 \% \mathrm{Nav} \alpha, 40 \% \mathrm{Nav} \beta, 40 \% \mathrm{EGFP})$ was transfected, except for the poison frog Navs for which a total of $3 \mu \mathrm{g}$ plasmid DNA (70\% Nava, 15\% EGFP, 15\% SV40 T antigen) was used to increase current amplitude. Experiments studying $R n$ Nav1.4 constructs were conducted using Chinese hamster ovary (CHO) cells cultured as described previously (54). Briefly, cells were grown in DMEM (GIBCO, Grand Island, NY) supplemented with $10 \%$ cosmic calf serum (HyClone, Logan, UT) and $100 \mathrm{U} / \mathrm{mL}$ penicillinstreptomycin (GIBCO). Cells were kept in a $5 \% \mathrm{CO}_{2}$ and $96 \%$ relative humidity incubator. $\mathrm{CHO}$ 
cells were transfected (in $10 \mathrm{~cm}$ plate) using the calcium phosphate precipitation method and EGFP expression as a marker of transfection.

$\mathrm{Na}^{+}$currents were recorded by whole cell patch clamp (73) at room temperature $\left(23 \pm 2{ }^{\circ} \mathrm{C}\right) 48-$ $72 \mathrm{~h}$ post-transfection. Data collection was performed using an Axopatch 200B amplifier (Molecular Devices) and pCLAMP 9 (Molecular Devices, Sunnyvale, CA, USA).

Pipettes were pulled from borosilicate glass capillaries (TW150F-3; World Precision Instruments, Sarasota, FL, USA) and polished with a microforge (MF-900; Narishige, Tokyo, Japan) to obtain 1.2-3.5 $\mathrm{M} \Omega$ resistances. Sixty to eighty percent of the voltage error due to the series resistance was compensated. For experiments with HEK 293 cells, pipette solution contained the following, in millimolar: 120, Cs methane sulfonate; 8, NaCl; 10, EGTA; 2, Mg-ATP; and 20, Hepes (pH 7.4 with $\mathrm{CsOH}$ ). Bath solution contained the following, in millimolar: $155, \mathrm{NaCl} ; 1, \mathrm{CaCl}$; $1, \mathrm{MgCl}$; $5, \mathrm{KCl} ; 10$, Hepes; and 10, glucose ( $\mathrm{pH} 7.4$ with $\mathrm{NaOH})$. For experiments with $\mathrm{CHO}$ cells, pipette solution contained the following, in millimolar: 125, CsCl; 40, NaF; 1, EDTA; and 20, Hepes (pH 7.4 with $\mathrm{CsOH}$ ). Bath solution contained the following, in millimolar: $160, \mathrm{NaCl} ; 2, \mathrm{CaCl}_{2}$; and 20 , Hepes (pH 7.4 with $\mathrm{NaOH})$.

For experiments with HEK 293 cells, voltage-dependent activation was assessed by stimulating the cells with a multistep depolarization protocol from -90 to $+50 \mathrm{mV}$ using $5 \mathrm{mV}$ increments, a $-100 \mathrm{mV}$ holding potential, and a sweep-to-weep interval duration of $2 \mathrm{~s}$. Voltage-dependent steady state inactivation was assessed by stimulating the cells with a 500 -ms pre-pulse depolarization from -110 to $0 \mathrm{mV}$ in $5 \mathrm{mV}$ steps, followed by a $20-\mathrm{ms}$ step to $0 \mathrm{mV}$, and repolarization to the holding potential, $-100 \mathrm{mV}$; sweep-to-sweep interval duration was $4 \mathrm{~s}$. To examine BTX effects, cells were stimulated upon BTX exposure by applying at least 120 step pulses from -120 to $0 \mathrm{mV}$ at $2 \mathrm{~Hz}$ frequency in order to facilitate BTX access into the channel pore as BTX is known to preferentially interact with the open state of $\mathrm{Na}_{v} \mathrm{~s}$ (74). For experiments with $\mathrm{CHO}$ cells, voltage-dependent activation was assessed by stimulating the cells with a multistep depolarization protocol from -120 to $+50 \mathrm{mV}$ using $5 \mathrm{mV}$ increments and a $-120 \mathrm{mV}$ holding potential. Voltage-dependent steady state inactivation was assessed by stimulating the cells with a 150 -ms pre-pulse depolarization from -140 to $0 \mathrm{mV}$ in $5 \mathrm{mV}$ steps, followed by a $50 \mathrm{~ms}$ step to $0 \mathrm{mV}$, and repolarization to the holding potential, $-120 \mathrm{mV}$. Equilibration of BTX was accomplished with persistent activation of channels by applying a $24 \mathrm{~ms}$ step depolarization from -120 to $0 \mathrm{mV}$ at a frequency of $2 \mathrm{~Hz}$ over the course of $8 \mathrm{~min}$. Leak currents were subtracted using a P/4 protocol during data acquisition. Data analysis was performed using Clampfit 10.6 (Axon Instruments). 
Activation curves were obtained by fitting the data with the following single or double Boltzmann equations: $\mathrm{I}=\operatorname{Imax} /(1+\exp ((\mathrm{V} 0.5-\mathrm{Vm}) / \mathrm{k}))$, or $\mathrm{I}=\left(\operatorname{Imax} 1 /\left(1+\exp \left(\left(\mathrm{V} 0.5_{1}-\mathrm{Vm}_{\mathrm{m}}\right) / \mathrm{k}_{1}\right)\right)\right)+$ $\left(\operatorname{Imax} 2 /\left(1+\exp \left(\left(V 0.5_{2}-\mathrm{Vm}\right) / \mathrm{k}_{2}\right)\right)\right)$, where Imax is the maximal current after normalization to the driving force, $\mathrm{V} 0.5$ is the half-activation potential, $\mathrm{Vm}$ is the membrane potential, and $\mathrm{k}$ is the slope factor. Inactivation curves were obtained by fitting the data with the following single Boltzmann equation: I = Imax/(1+exp((Vm-V0.5)/k)), where Imax is the absolute value of the maximal current at the test pulse, V0.5 is the half-inactivation potential, $\mathrm{Vm}$ is the membrane potential, and $\mathrm{k}$ is the slope factor. Current density was determined as the ratio between current amplitude $(\mathrm{pA})$ and the membrane capacitance $(\mathrm{pF})$.

\section{Two-electrode voltage clamp electrophysiology}

Two-electrode voltage-clamp recordings were performed on defolliculated stage V-VI Xenopus laevis oocytes harvested under UCSF-IACUC protocol AN178461 1-2 days after microinjection with mRNA. Linearized Pitohui (Pk), P. terribilis (Pt), or D. tinctorius (Dt) Nav1.4 cDNA was translated into capped mRNA using the mMESSAGE mMACHINE ${ }^{\text {TM }}$ T7 Transcription Kit (Invitrogen). Xenopus oocytes were injected with 0.5-2 ng, 3-6 ng or 10-30 ng of Pk Nav1.4, Pt Nav1.4 or Dt Nav1.4 mRNA, respectively. Two-electrode voltage clamp experiments were performed 1-2 days post-injection. Data were acquired using a GeneClamp 500B amplifier (MDS Analytical Technologies) controlled by pClamp software (Molecular Devices), and digitized at $1 \mathrm{kHz}$ using Digidata 1332A digitizer (MDS Analytical Technologies).

Oocytes were impaled with borosilicate recording microelectrodes (0.3-3.0 $\mathrm{M} \Omega$ resistance) backfilled with $3 \mathrm{M} \mathrm{KCl}$. Sodium currents were recorded using a bath solution containing the following, in millimolar: $96, \mathrm{NaCl} ; 1, \mathrm{CaCl}_{2} ; 1, \mathrm{MgCl}_{2} ; 2, \mathrm{KCl}$; and 5, $\mathrm{HEPES}(\mathrm{pH} 7.5$ with $\mathrm{NaOH})$, supplemented with antibiotics $\left(50 \mu \mathrm{g} \mathrm{ml}^{-1}\right.$ gentamycin, $100 \mathrm{IU} \mathrm{ml}^{-1}$ penicillin and $100 \mu \mathrm{g} \mathrm{ml}^{-1}$ streptomycin) and $2.5 \mathrm{mM}$ sodium pyruvate.

For studying the competition between tricaine and BTX, $0.5 \mathrm{mM}$ tricaine was applied by continuous perfusion in the bath solution to assess channel block. BTX was applied from the intracellular side of the membrane by injecting oocytes with $50 \mathrm{~nL}$ of $2 \mathrm{mM} \mathrm{BTX}$. Following BTX injection, oocytes were stimulated by applying 1000 step pulses of $60 \mathrm{~ms}$ each, from -120 to 0 $\mathrm{mV}$ at $2 \mathrm{~Hz}$ frequency in order to facilitate BTX access into the channel pore.

To determine STX and TTX dose-response curves, solutions containing test concentrations of each toxin were applied in series by perfusion to oocytes expressing Pk Nav1.4, Pt Nav1.4, or Dt Nav1.4. $I C_{50}$ values were calculated from the ratio of peak currents in the presence and absence 
of toxin, based on the following equation: $\frac{I_{x}}{I_{0}}=\frac{\left(I_{\max }-I_{\min }\right)}{\left(1+\frac{x}{I C_{50}}\right)}$ where $I_{\mathrm{x}}$ is the current amplitude at the toxin concentration $x, I_{0}$ is the current amplitude in absence of toxin, $I_{\max }$ and $I_{\min }$ are the maximum and minimum peak current amplitudes, respectively, and $\mathrm{IC}_{50}$ is the half-maximal inhibitory concentration.

All toxin effects were assessed with 60 -ms depolarization steps from -120 to $0 \mathrm{mV}$ with a holding potential of $-120 \mathrm{mV}$ and a sweep-to-sweep duration of $10 \mathrm{~s}$.

Recordings were conducted at room temperature $\left(23 \pm 2{ }^{\circ} \mathrm{C}\right)$. Leak currents were subtracted using a P/4 protocol during data acquisition. Data Analysis was performed using Clampfit 10.6 (Axon Instruments) and a custom software developed in the Igor environment (Wavemetrics).

\section{Toxin challenge experiments}

Frogs for the toxin challenge experiments were obtained from the following sources: Polypedates leucomystax, Phyllobates terribilis, and Dendrobates tinctorius (Josh's frogs, Owosso, MI, USA); Xenopus laevis (Nasco); and Mantella aurantiaca (Indoor Ecosystems, Whitehouse, $\mathrm{OH}$, USA). All experiments were performed in accordance with UCSF IACUC protocol AN136799.

Frogs were held at room temperature $\left(23 \pm 2{ }^{\circ} \mathrm{C}\right)$ and anesthetized with a $0.15 \%$ tricaine (MS-222) bath in prior to toxin injections. Once under anesthesia, as judged by immobility and lack of response to foot pinching, frogs were weighed in order to calculate the appropriate amount of toxin to be administered to at $20 x$ the $L_{50}$ based on the values calculated for mice as follows: BTX: $2 \mu \mathrm{g}$ per kg, (39), STX: $10 \mu \mathrm{g}$ per kg (75), and TTX: $12.5 \mu \mathrm{g}$ per kg, (76). BTX, STX, and TTX, were delivered $40 \mathrm{ng}, 200 \mathrm{ng}$, and $250 \mathrm{ng}$ of toxin, respectively, per $\mathrm{g}$ of animal weight. The upper right hind leg was injected with either control, phosphate-buffered saline (PBS), or toxin containing solutions under an SMZ645 binocular microscope (Nikon) using a 30G PrecisionGlideTM needle (BD, Franklin Lakes, NJ, USA). Batrachotoxin (BTX), saxitoxin (STX) or tetrodotoxin (TTX) dissolved in PBS were injected at the appropriate concentration to deliver 20 times the $\mathrm{LD}_{50}$. The total volume of injection was $100 \mu \mathrm{L}$ in $X$. laevis and $10 \mu \mathrm{L}$ in other frogs due to their smaller size. The choice of intramuscular injection was to avoid internal organ damage. Frogs were allowed to recover in a separate container and monitored constantly for signs of recovery, paralysis, or other adverse symptoms. For $X$. laevis, the recovery container was filled with deionized water and inclined in a way that the frogs could recover on the dry surface of the container base. Post-recovery activity was then assessed by the ability of the frogs to move from the dry to the water-containing surface as $X$. laevis are primarily aquatic animals. For all other frogs, post-recovery activity was assessed by monitoring the ability of the animals to put 
bioRxiv preprint doi: https://doi.org/10.1101/2020.10.29.361212; this version posted October 29, 2020. The copyright holder for this preprint (which was not certified by peer review) is the author/funder, who has granted bioRxiv a license to display the preprint in perpetuity. It is made available under aCC-BY-NC-ND 4.0 International license.

themselves right-side up from a supine position in their recovery containers. The monitoring period was up to 24 hours post injection. 


\section{References}

1. A. H. Savitzky et al., Sequestered defensive toxins in tetrapod vertebrates: principles, patterns, and prospects for future studies. Chemoecology 22, 141-158 (2012).

2. J. C. Santos, R. D. Tarvin, L. A. O'Connell, in Chemical Signals in Vertebrates 13. (Springer International Publishing, Switzerland, 2016), vol. 13, pp. 305-337.

3. J. P. Dumbacher, B. M. Beehler, T. F. Spande, H. M. Garraffo, J. W. Daly, Homobatrachotoxin in the genus Pitohui: chemical defense in birds? Science 258, 799801 (1992).

4. J. P. Dumbacher, T. F. Spande, J. W. Daly, Batrachotoxin alkaloids from passerine birds: a second toxic bird genus (Ifrita kowaldi) from New Guinea. Proc Natl Acad Sci U S A 97, 12970-12975 (2000).

5. J. W. Daly et al., An uptake system for dietary alkaloids in poison frogs (Dendrobatidae). Toxicon 32, 657-663 (1994).

6. J. W. Daly, H. M. Garraffo, T. F. Spande, C. Jaramillo, A. S. Rand, Dietary Source for Skin Alkaloids of Poison Frogs (Dendrobatidae). Journal of Chemical Ecology 20, 943-955 (1994).

7. J. P. Dumbacher et al., Melyrid beetles (Choresine): a putative source for the batrachotoxin alkaloids found in poison-dart frogs and toxic passerine birds. Proc Natl Acad Sci U S A 101, 15857-15860 (2004).

8. J. P. Dumbacher, G. K. Menon, J. W. Daly, Skin as a Toxin Storage Organ in the Endemic New Guinean Genus Pitohui. Auk 126, 520-530 (2009).

9. W. A. Catterall, Activation of the action potential Na+ ionophore by neurotoxins. An allosteric model. J Biol Chem 252, 8669-8676 (1977).

10. B. I. Khodorov, Batrachotoxin as a tool to study voltage-sensitive sodium channels of excitable membranes. Prog Biophys Mol Biol 45, 57-148 (1985).

11. M. M. Logan, T. Toma, R. Thomas-Tran, J. Du Bois, Asymmetric synthesis of batrachotoxin: Enantiomeric toxins show functional divergence against NaV. Science 354, 865-869 (2016).

12. S. Y. Wang, G. K. Wang, Voltage-gated sodium channels as primary targets of diverse lipid-soluble neurotoxins. Cell Signal 15, 151-159 (2003).

13. K. Arbuckle, R. C. Rodriguez de la Vega, N. R. Casewell, Coevolution takes the sting out of it: Evolutionary biology and mechanisms of toxin resistance in animals. Toxicon 140, 118-131 (2017).

14. K. H. Almabruk, L. K. Dinh, B. Philmus, Self-Resistance of Natural Product Producers: Past, Present, and Future Focusing on Self-Resistant Protein Variants. ACS Chem Biol 13, 1426-1437 (2018).

15. P. Hunter, Do not poison thyself Mechanisms to avoid self-toxicity could inspire novel compounds and pathways for synthetic biology and applications for agriculture. Embo Rep 19, (2018).

16. R. D. Tarvin et al., Interacting amino acid replacements allow poison frogs to evolve epibatidine resistance. Science 357, 1261-1266 (2017).

17. V. M. Bricelj et al., Sodium channel mutation leading to saxitoxin resistance in clams increases risk of PSP. Nature 434, 763-767 (2005).

18. C. T. Hanifin, W. F. Gilly, Evolutionary history of a complex adaptation: Tetrodotoxin resistance in salamanders. Evolution 69, 232-244 (2015).

19. M. C. Jost et al., Toxin-resistant sodium channels: parallel adaptive evolution across a complete gene family. Mol Biol Evol 25, 1016-1024 (2008).

20. S. Geffeney, E. D. Brodie, Jr., P. C. Ruben, E. D. Brodie, 3rd, Mechanisms of adaptation in a predator-prey arms race: TTX-resistant sodium channels. Science 297, 1336-1339 (2002). 
21. S. L. Geffeney, E. Fujimoto, E. D. Brodie, 3rd, E. D. Brodie, Jr., P. C. Ruben, Evolutionary diversification of TTX-resistant sodium channels in a predator-prey interaction. Nature 434, 759-763 (2005).

22. J. W. McGlothlin et al., Historical Contingency in a Multigene Family Facilitates Adaptive Evolution of Toxin Resistance. Curr Biol 26, 1616-1621 (2016).

23. J. W. Daly, C. W. Myers, J. E. Warnick, E. X. Albuquerque, Levels of batrachotoxin and lack of sensitivity to its action in poison-dart frogs (Phyllobates). Science 208, 1383-1385 (1980).

24. R. D. Tarvin, J. C. Santos, L. A. O'Connell, H. H. Zakon, D. C. Cannatella, Convergent Substitutions in a Sodium Channel Suggest Multiple Origins of Toxin Resistance in Poison Frogs. Mol Biol Evol 33, 1068-1081 (2016).

25. S. Y. Wang, G. K. Wang, Single rat muscle $\mathrm{Na}(+)$ channel mutation confers batrachotoxin autoresistance found in poison-dart frog Phyllobates terribilis. Proc Natl Acad Sci U S A 114, 10491-10496 (2017).

26. R. Marquez, V. Ramirez-Castaneda, A. Amezquita, Does batrachotoxin autoresistance coevolve with toxicity in Phyllobates poison-dart frogs? Evolution 73, 390-400 (2019).

27. E. X. Albuquerque, J. E. Warnick, F. M. Sansone, J. Daly, The pharmacology of batrachotoxin. V. A comparative study of membrane properties and the effect of batrachotoxin on sartorius muscles of the frogs Phyllobates aurotaenia and Rana pipiens. J Pharmacol Exp Ther 184, 315-329 (1973).

28. C. R. Feldman et al., Is there more than one way to skin a newt? Convergent toxin resistance in snakes is not due to a common genetic mechanism. Heredity (Edinb) 116, 84-91 (2016).

29. M. T. J. Hague et al., Large-effect mutations generate trade-off between predatory and locomotor ability during arms race coevolution with deadly prey. Evol Lett 2, 406-416 (2018).

30. L. E. Llewellyn, P. M. Bell, E. G. Moczydlowski, Phylogenetic survey of soluble saxitoxinbinding activity in pursuit of the function and molecular evolution of saxiphilin, a relative of transferrin. Proc Biol Sci 264, 891-902 (1997).

31. J. Mahar, G. L. Lukacs, Y. Li, S. Hall, E. Moczydlowski, Pharmacological and biochemical properties of saxiphilin, a soluble saxitoxin-binding protein from the bullfrog (Rana catesbeiana). Toxicon 29, 53-71 (1991).

32. G. K. Menon, J. P. Dumbacher, A 'toxin mantle' as defensive barrier in a tropical bird: evolutionary exploitation of the basic permeability barrier forming organelles. Exp Dermatol 23, 288-290 (2014).

33. W. A. Catterall, M. J. Lenaeus, T. M. Gamal El-Din, Structure and Pharmacology of Voltage-Gated Sodium and Calcium Channels. Annu Rev Pharmacol Toxicol 60, 133-154 (2020).

34. Y. Du, D. P. Garden, L. Wang, B. S. Zhorov, K. Dong, Identification of new batrachotoxinsensing residues in segment IIIS6 of the sodium channel. J Biol Chem 286, 13151-13160 (2011).

35. J. D. Calhoun, L. L. Isom, The role of non-pore-forming beta subunits in physiology and pathophysiology of voltage-gated sodium channels. Handb Exp Pharmacol 221, 51-89 (2014).

36. J. Gilchrist, B. M. Olivera, F. Bosmans, Animal toxins influence voltage-gated sodium channel function. Handb Exp Pharmacol 221, 203-229 (2014).

37. M. M. Zhang et al., Co-expression of $\mathrm{Na}(\mathrm{V})$ beta subunits alters the kinetics of inhibition of voltage-gated sodium channels by pore-blocking mu-conotoxins. $\mathrm{Br} J$ Pharmacol 168, 1597-1610 (2013).

38. S. Das, J. Gilchrist, F. Bosmans, F. Van Petegem, Binary architecture of the Nav1.2-beta2 signaling complex. eLife 5, (2016). 
39. E. X. Albuquerque, J. W. Daly, B. Witkop, Batrachotoxin: chemistry and pharmacology. Science 172, 995-1002 (1971).

40. C. W. Myers, J. W. Daly, B. Malkin, A dangerously toxic new frog (Pyllobates) used by Emberà Indians of Western Colombia, with discusison of blowgun fabrication and dart poisoning. Bulletin of the American Museum of Natural History 161, 309-365 (1978).

41. G. K. Wang, C. Quan, S. Wang, A common local anesthetic receptor for benzocaine and etidocaine in voltage-gated mu1 Na+ channels. Pflugers Arch 435, 293-302 (1998).

42. M. F. Sheets, H. A. Fozzard, D. A. Hanck, Important Role of Asparagines in Coupling the Pore and Votage-Sensor Domain in Voltage-Gated Sodium Channels. Biophys $J 109$, 2277-2286 (2015).

43. X. Pan et al., Structure of the human voltage-gated sodium channel Nav1.4 in complex with beta1. Science 362, (2018).

44. J. W. Daly, T. F. Spande, H. M. Garraffo, Alkaloids from amphibian skin: a tabulation of over eight-hundred compounds. J Nat Prod 68, 1556-1575 (2005).

45. J. W. Daly et al., Individual and geographic variation of skin alkaloids in three species of Madagascan poison frogs (Mantella). J Chem Ecol 34, 252-279 (2008).

46. H. M. Garraffo et al., Alkaloids in Madagascan frogs (Mantella): pumiliotoxins, indolizidines, quinolizidines, and pyrrolizidines. J Nat Prod 56, 1016-1038 (1993).

47. A. P. Thottumkara, W. H. Parsons, J. Du Bois, Saxitoxin. Angew Chem Int Ed Engl 53, 5760-5784 (2014).

48. L. M. Duran-Riveroll, A. D. Cembella, Guanidinium Toxins and Their Interactions with Voltage-Gated Sodium Ion Channels. Mar Drugs 15, (2017).

49. R. K. Finol-Urdaneta et al., Batrachotoxin acts as a stent to hold open homotetrameric prokaryotic voltage-gated sodium channels. J Gen Physiol 151, 186-199 (2019).

50. G. K. Wang, S. Y. Wang, Inactivation of batrachotoxin-modified Na+ channels in GH3 cells. Characterization and pharmacological modification. J Gen Physiol 99, 1-20 (1992).

51. G. K. Wang, W. M. Mok, S. Y. Wang, Charged tetracaine as an inactivation enhancer in batrachotoxin-modified Na+ channels. Biophys J 67, 1851-1860 (1994).

52. G. K. Wang, S. Y. Wang, Binding of benzocaine in batrachotoxin-modified $\mathrm{Na}$ + channels. State-dependent interactions. J Gen Physiol 103, 501-518 (1994).

53. R. Thomas-Tran, J. Du Bois, Mutant cycle analysis with modified saxitoxins reveals specific interactions critical to attaining high-affinity inhibition of hNaV1.7. Proc Natl Acad Sci U S A 113, 5856-5861 (2016).

54. B. M. Andresen, J. Du Bois, De novo synthesis of modified saxitoxins for sodium ion channel study. J Am Chem Soc 131, 12524-12525 (2009).

55. M. Chahine, P. B. Bennett, A. L. George, Jr., R. Horn, Functional expression and properties of the human skeletal muscle sodium channel. Pflugers Arch 427, 136-142 (1994).

56. V. L. Thomas, A. C. McReynolds, B. K. Shoichet, Structural bases for stability-function tradeoffs in antibiotic resistance. J Mol Biol 396, 47-59 (2010).

57. X. Wang, G. Minasov, B. K. Shoichet, Evolution of an antibiotic resistance enzyme constrained by stability and activity trade-offs. J Mol Biol 320, 85-95 (2002).

58. L. S. Bigman, Y. Levy, Proteins: molecules defined by their trade-offs. Curr Opin Struct Biol 60, 50-56 (2020).

59. N. Tokuriki, F. Stricher, L. Serrano, D. S. Tawfik, How protein stability and new functions trade off. PLoS Comput Biol 4, e1000002 (2008).

60. C. Chen et al., Reduced sodium channel density, altered voltage dependence of inactivation, and increased susceptibility to seizures in mice lacking sodium channel beta 2-subunits. Proc Natl Acad Sci U S A 99, 17072-17077 (2002).

61. G. Loussouarn et al., Physiological and Pathophysiological Insights of Nav1.4 and Nav1.5 Comparison. Frontiers in pharmacology 6, 314 (2015). 
62. C. H. Lee, D. K. Jones, C. Ahern, M. F. Sarhan, P. C. Ruben, Biophysical costs associated with tetrodotoxin resistance in the sodium channel pore of the garter snake, Thamnophis sirtalis. J Comp Physiol A Neuroethol Sens Neural Behav Physiol 197, 33-43 (2011).

63. M. Prinzmetal, H. Sommer, C. D. Leake, The pharmacological action of "mussel poison". J Pharmacol Exp Ther 46, 63-73 (1932).

64. C. Y. Kao, F. A. Fuhrman, Differentiation of Actions of Tetrodotoxin and Saxitoxin. Toxicon 5, 25-34 (1967).

65. T.-J. Yen, M. Lolicato, R. Thomas-Tran, J. Du Bois, D. L. Minor, Jr., Structure of the Saxiphilin:saxitoxin (STX) complex reveals a convergent molecular recognition strategy for paralytic toxins. Sci Adv 5, (2019).

66. S. N. Caty et al., Molecular physiology of chemical defenses in a poison frog. J Exp Biol 222, (2019).

67. L. A. O'Connell et al., Rapid toxin squestration impacts poison frog physiology. http://www.biorxiv.org/content/10.1101/2020.05.27.119081v1, (2020).

68. M. Yotsu-Yamashita et al., Purification, characterization, and cDNA cloning of a novel soluble saxitoxin and tetrodotoxin binding protein from plasma of the puffer fish, Fugu pardalis. Eur J Biochem 268, 5937-5946 (2001).

69. M. Yotsu-Yamashita, H. Yamaki, N. Okoshi, N. Araki, Distribution of homologous proteins to puffer fish saxitoxin and tetrodotoxin binding protein in the plasma of puffer fish and among the tissues of Fugu pardalis examined by Western blot analysis. Toxicon 55, 11191124 (2010).

70. A. M. Bolger, M. Lohse, B. Usadel, Trimmomatic: a flexible trimmer for Illumina sequence data. Bioinformatics 30, 2114-2120 (2014).

71. A. Bankevich et al., SPAdes: a new genome assembly algorithm and its applications to single-cell sequencing. J Comput Biol 19, 455-477 (2012).

72. J. G. Ruby, P. Bellare, J. L. Derisi, PRICE: software for the targeted assembly of components of (Meta) genomic sequence data. G3 (Bethesda) 3, 865-880 (2013).

73. O. P. Hamill, A. Marty, E. Neher, B. Sakmann, F. J. Sigworth, Improved patch-clamp techniques for high-resolution current recording from cells and cell-free membrane patches. Pflugers Arch 391, 85-100 (1981).

74. J. Tanguy, J. Z. Yeh, BTX modification of Na channels in squid axons. I. State dependence of BTX action. J Gen Physiol 97, 499-519 (1991).

75. G. S. Wiberg, N. R. Stephenson, Toxicologic studies on paralytic shellfish poison. Toxicol Appl Pharmacol 2, 607-615 (1960).

76. J. Lago, L. P. Rodriguez, L. Blanco, J. M. Vieites, A. G. Cabado, Tetrodotoxin, an Extremely Potent Marine Neurotoxin: Distribution, Toxicity, Origin and Therapeutical Uses. Mar Drugs 13, 6384-6406 (2015).

77. Z. Yan et al., Structure of the Nav1.4-beta1 Complex from Electric Eel. Cell 170, 470-482 e411 (2017). 


\section{Table 1 Activation parameters}

\begin{tabular}{|c|c|c|c|c|c|c|c|c|}
\hline \multicolumn{2}{|c|}{ Channel } & \multirow{2}{*}{$\begin{array}{l}\begin{array}{l}\text { Current } \\
\text { density }\end{array} \\
119.5 \pm 11.8\end{array}$} & \multirow{2}{*}{$\begin{array}{l}V_{1 / 2}-\mathrm{I} \\
-23.4 \pm 1.0\end{array}$} & \multirow[t]{2}{*}{$\Delta \mathrm{V}_{1 / 2 \mathrm{BTX}}$} & \multirow{2}{*}{$\begin{array}{l}\mathrm{k}_{\text {act }} \mathrm{I} \\
5.9 \pm 0.3\end{array}$} & \multirow[t]{2}{*}{$\mathrm{V}_{1 / 2}-\mathrm{II}$} & \multirow[t]{2}{*}{$\mathrm{k}_{\mathrm{act}}-\mathrm{II}$} & \multirow{2}{*}{$\begin{array}{l}\mathrm{n} \\
20\end{array}$} \\
\hline \multirow{16}{*}{ ? } & Pum Nav1.4 & & & & & & & \\
\hline & $+\mathrm{BTX}$ & & $-57.0 \pm 0.7$ & $-33.6 \pm 1.2$ & $3.8 \pm 0.6$ & $-26.0 \pm 1.7$ & $5.9 \pm 0.8$ & 9 \\
\hline & Pum Nav1.4 + PkNavß2 & $86.5 \pm 12.8$ & $-24.9 \pm 1.3$ & & $6.2 \pm 0.5$ & & & 15 \\
\hline & $+\mathrm{BTX}$ & & $-59.9 \pm 1.9$ & $-35.0 \pm 2.3$ & $3.5 \pm 0.5$ & $-28.0 \pm 1.4$ & $5.7 \pm 0.6$ & 7 \\
\hline & Pum Nav1.5 & $17.1 \pm 3.5$ & $-22.4 \pm 1.6$ & & $8.6 \pm 0.3$ & & & 9 \\
\hline & $+\mathrm{BTX}$ & & $-59.8 \pm 0.8$ & $-37.4 \pm 1.8$ & $5.5 \pm 1.9$ & $-25.0 \pm 2.3$ & $11.3 \pm 0.7$ & 8 \\
\hline & Pum Nav1.4 N432T (DI) & $107.5 \pm 26.9$ & $-20.2 \pm 1.9$ & & $6.7 \pm 0.5$ & & & 7 \\
\hline & +BTX & & $-51.0 \pm 0.9$ & $-30.8 \pm 2.1$ & $4.4 \pm 0.4$ & $-9.9 \pm 6.1$ & $7.4 \pm 1.4$ & 10 \\
\hline & Pum Nav1.4 N830T (DII) & $8.1 \pm 1.5$ & $-22.4 \pm 1.0$ & & $9.2 \pm 0.5$ & & & 16 \\
\hline & $+\mathrm{BTX}$ & & $-54.0 \pm 1.7$ & $-31.6 \pm 2.0$ & $7.6 \pm 1.0$ & $-22.9 \pm 1.8$ & $5.2 \pm 0.9$ & 9 \\
\hline & Pum Nav1.4 N1306T (DIII) & $103.7 \pm 29.4$ & $-26.7 \pm 1.0$ & & $6.4 \pm 0.3$ & & & 10 \\
\hline & $+\mathrm{BTX}$ & & $-63.6 \pm 1.0$ & $-36.9 \pm 1.4$ & $3.7 \pm 0.6$ & $-24.8 \pm 1.2$ & $8.5 \pm 1.3$ & 5 \\
\hline & Pum Nav1.4 N1609T (DIV) & $24.6 \pm 10.8$ & $-2.9 \pm 1.1$ & & $9.6 \pm 1.0$ & & & 9 \\
\hline & $+\mathrm{BTX}$ & & $-14.3 \pm 3.0$ & $-11.4 \pm 3.2$ & $9.7 \pm 2.1$ & N/A & N/A & 5 \\
\hline & Pum Nav1.4 N1609A (DIV) & $28.9 \pm 4.6$ & $-3.8 \pm 1.3$ & & $10.7 \pm 0.4$ & & & 21 \\
\hline & $+\mathrm{BTX}$ & & $-45.1 \pm 2.2$ & $-41.3 \pm 2.6$ & $9.1 \pm 0.8$ & $-9.6 \pm 2.0$ & $8.6 \pm 1.1$ & 14 \\
\hline \multirow{4}{*}{ 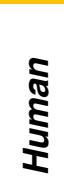 } & Hs Nav1.4 & $70.9 \pm 13.8$ & $-13.3 \pm 1.6$ & & $6.1 \pm 0.4$ & & & 13 \\
\hline & $+\mathrm{BTX}$ & & $-49.2 \pm 0.9$ & $-35.9 \pm 1.8$ & $5.0 \pm 0.4$ & $-19.0 \pm 1.9$ & $5.2 \pm 0.5$ & 9 \\
\hline & Hs Nav1.4 N1591T (DIV) & $31.7 \pm 13.4$ & $3.6 \pm 2.2$ & & $10.9 \pm 1.1$ & & & 4 \\
\hline & $+\mathrm{BTX}$ & & $-10.6 \pm 5.0$ & $-14.2 \pm 5.5$ & $9.7 \pm 0.6$ & N/A & $\mathrm{N} / \mathrm{A}$ & 6 \\
\hline \multirow{4}{*}{$\frac{\pi}{\pi}$} & Rn Nav1.4 & $445.0 \pm 125.1$ & $-23.1 \pm 0.3$ & & $7.3 \pm 0.3$ & & & 4 \\
\hline & $+\mathrm{BTX}$ & & $-68.7 \pm 0.2$ & $-45.6 \pm 0.4$ & $4.0 \pm 0.1$ & & & 4 \\
\hline & Rn Nav1.4 N1584T (DiV) & $43.2 \pm 40.1$ & $-5.1 \pm 0.5$ & & $8.6 \pm 0.3$ & & & 6 \\
\hline & $+\mathrm{BTX}$ & & $-45.4 \pm 2.7$ & $-40.3 \pm 2.8$ & $9.6 \pm 1.1$ & $-17.0 \pm 1.2$ & $13.0 \pm 0.9$ & 6 \\
\hline \multirow{12}{*}{$\begin{array}{l}0 \\
0 \\
2 \\
\delta \\
\delta \\
0 \\
0\end{array}$} & Pt $\mathrm{Na}_{\mathrm{v}} 1.4$ & $26.2 \pm 5.9$ & $-24.3 \pm 1.4$ & & $7.6 \pm 0.3$ & & & 6 \\
\hline & Oocytes & $669.7 \pm 120.4$ & $-16.2 \pm 2.0$ & & $4.3 \pm 0.6$ & & & 11 \\
\hline & $+\mathrm{BTX}$ & & $-54.3 \pm 1.6$ & $-30.0 \pm 2.1$ & $6.3 \pm 0.8$ & $-20.0 \pm 2.2$ & $9.1 \pm 1.5$ & 9 \\
\hline & +BTX oocytes & & $-49.3 \pm 2.5$ & $-33.1 \pm 3.2$ & $4.8 \pm 0.7$ & & & 8 \\
\hline & Pt Nav1.4 (N1600T) (DIV) & $24.1 \pm 3.9$ & $-23.8 \pm 1.2$ & & $9.0 \pm 0.3$ & & & 10 \\
\hline & $+\mathrm{BTX}$ & & $-54.6 \pm 2.2$ & $-30.8 \pm 2.5$ & $5.6 \pm 0.8$ & $-19.0 \pm 6.7$ & $11.7 \pm 2.0$ & 6 \\
\hline & Dt Nav1.4 & $11.1 \pm 1.8$ & $-17.1 \pm 1.6$ & & $7.7 \pm 0.4$ & & & 13 \\
\hline & oocytes & $108.1 \pm 24.3$ & $-19.6 \pm 2.5$ & & $5.1 \pm 0.6$ & & & 10 \\
\hline & + BTX & & $-55.0 \pm 1.2$ & $i-37.9 \pm 2.0$ & $5.8 \pm 1.4$ & $-17.4 \pm 2.7$ & $8.2 \pm 0.8$ & 6 \\
\hline & +BTX oocytes & & $-57.4 \pm 5.6$ & i-37.8 \pm 6.1 & $5.2 \pm 0.7$ & & & 5 \\
\hline & Dt Nav1.4 (N1600T) (DIV) & $10.0 \pm 2.0$ & $-17.2 \pm 1.2$ & & $10.7 \pm 0.9$ & & & $<$ \\
\hline & $+\mathrm{BTX}$ & & $-55.1 \pm 1.4$ & $-37.8 \pm 1.8$ & $6.0 \pm 0.5$ & $-18.0 \pm 3.1$ & $9.8 \pm 1.3$ & 4 \\
\hline
\end{tabular}




\section{Figure 1}
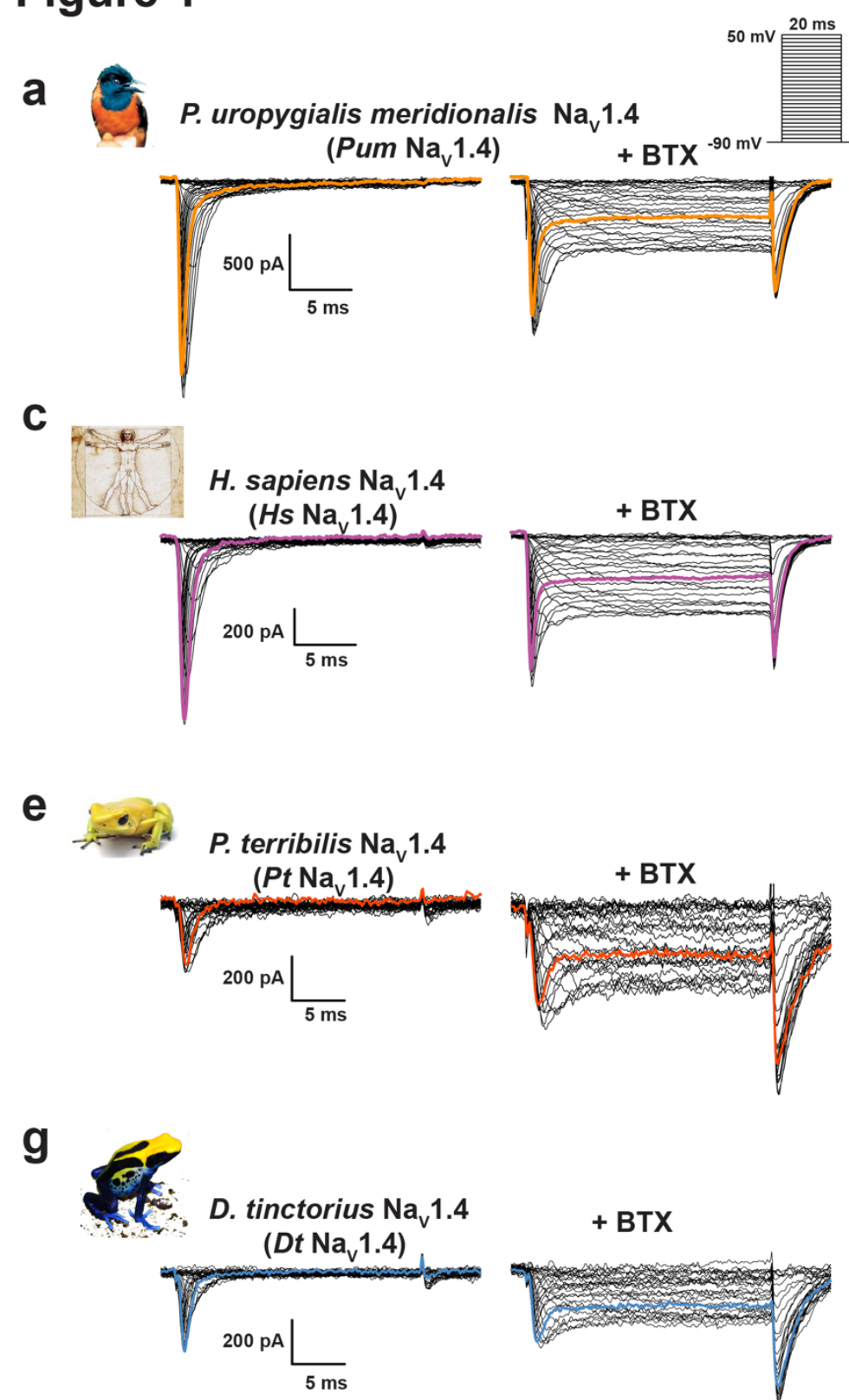

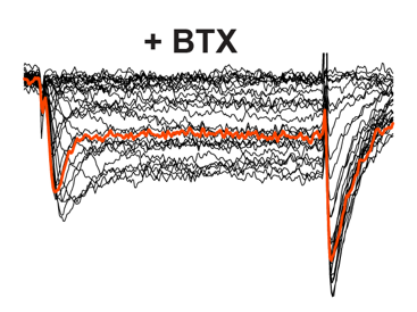

f
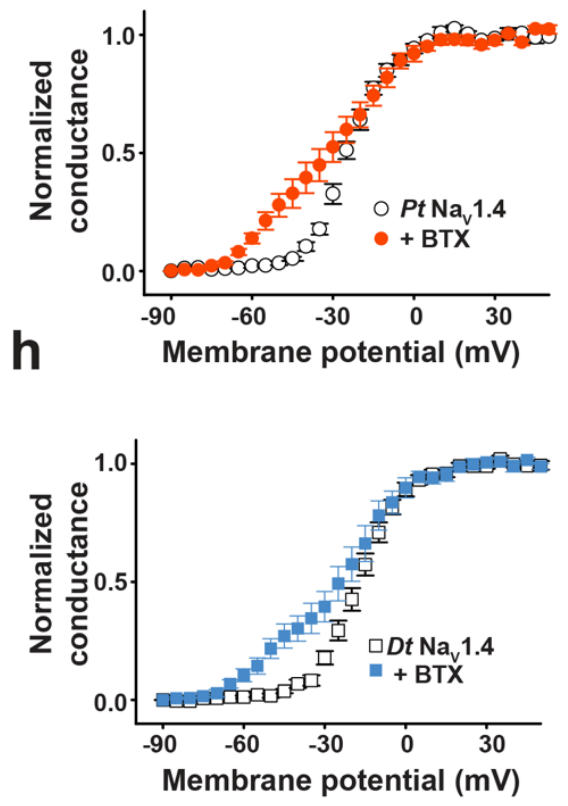

Figure 1 Pitohui and Poison frog Nav1.4 channels are BTX sensitive Exemplar current recordings (a, c, e, and g) for: a, Pitohui uropygialis meridionalis Nav1.4 (Pum Nav1.4); c, Human Nav1.4 (Hs Nav1.4); e, Phyllobates terribilis Nav1.4 (Pt Nav1.4); and g, Dendrobates tinctorius Nav1.4 (Dt Nav1.4) expressed in HEK293 cells in absence (left) or presence of $10 \mu \mathrm{M}$ BTX (right). Trace at $0 \mathrm{mV}$ is highlighted in each panel. Currents were evoked with the shown multistep 
depolarization protocol (inset panel 'a'). Conductance-voltage relationships (b, d, f, and $\mathbf{h}$ ) in presence or absence of $10 \mu \mathrm{M}$ BTX for b, Pum Nav1.4 (black diamonds), +BTX (orange diamonds), d, Hs Nav1.4 (black circles), +BTX (purple circles) f, Pt Nav1.4 (white circles), +BTX (orange circles), and h, Dt Nav1.4 (white squares), +BTX (blue squares). 
Figure 2

a

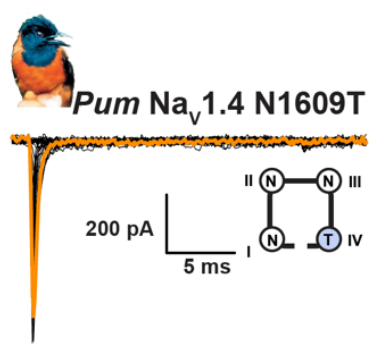

C

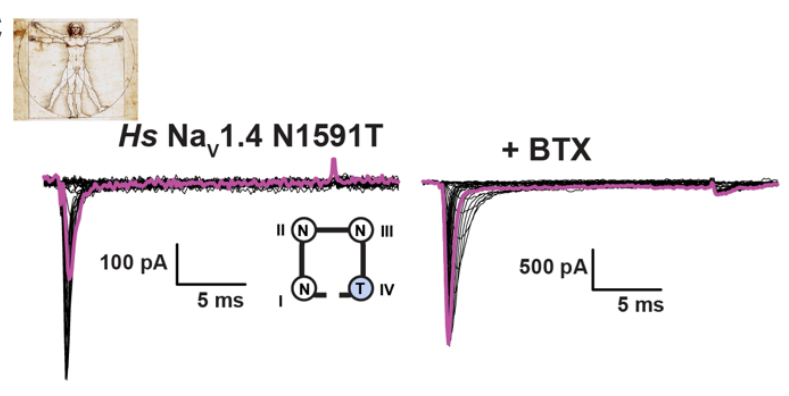

e
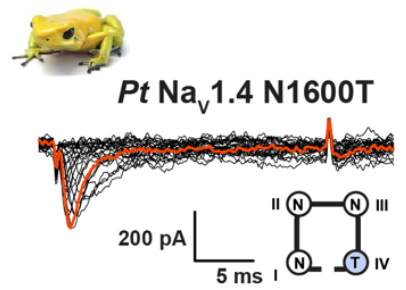

gef.
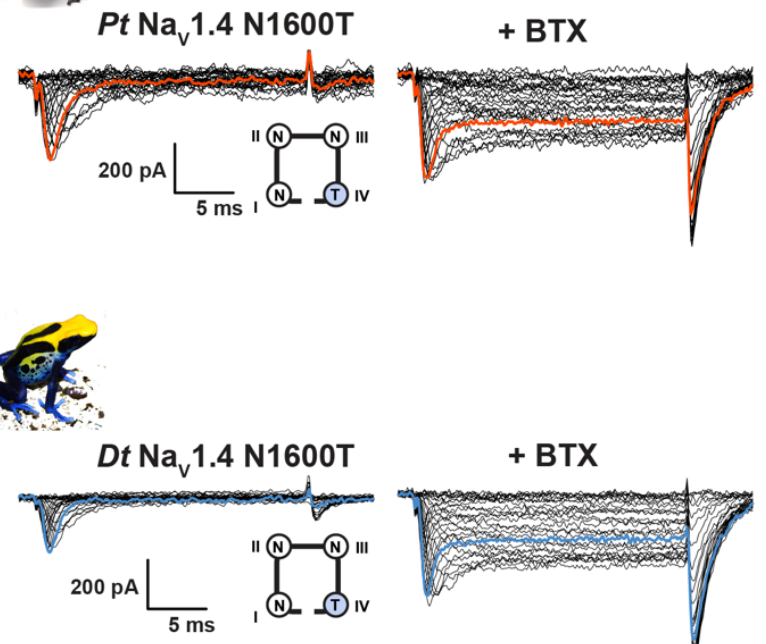

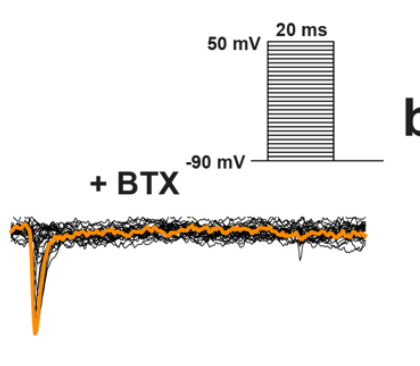

d
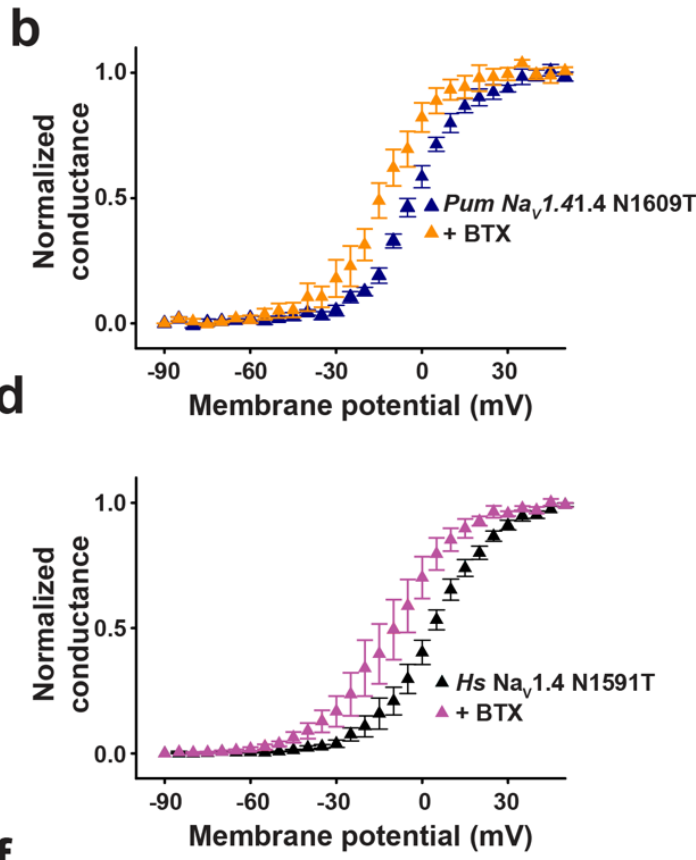

f

h
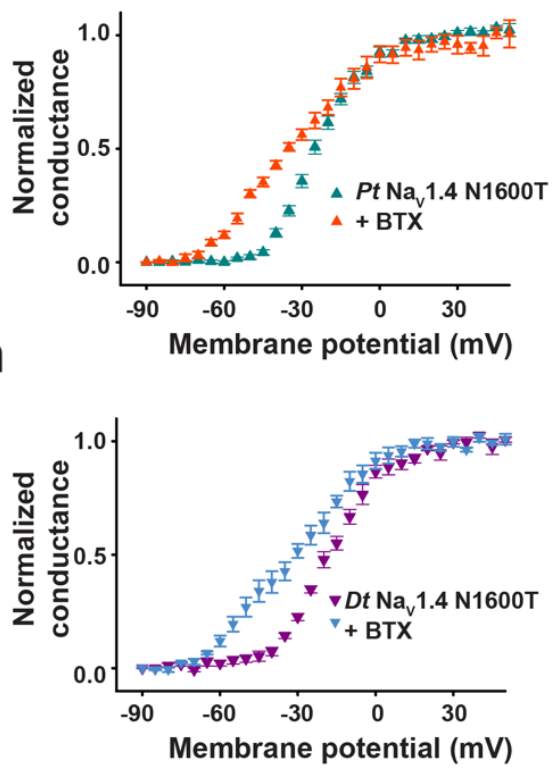

Figure 2. DIVS6 $\mathrm{N} \rightarrow \mathrm{T}$ mutation reduces BTX sensitivity of Pitohui and human but not poison frog Nav1.4s Exemplar current recordings (a, c, e, and g) for: a, Pum Nav1.4 N1609T; c, Hs Nav1.4 N1591T; e, Pt Nav1.4 N1600T ; and g, Dt Nav1.4 N1600T expressed in HEK293 cells in absence (left) or presence of $10 \mu \mathrm{M}$ BTX (right). Trace at $0 \mathrm{mV}$ is highlighted in each panel. Currents were evoked with the shown multistep depolarization protocol (inset panel ' $a$ '). Cartoon shows a diagram of the identities of the S6 'Asn' for each construct. Conductance-voltage 
bioRxiv preprint doi: https://doi.org/10.1101/2020.10.29.361212; this version posted October 29, 2020. The copyright holder for this preprint (which was not certified by peer review) is the author/funder, who has granted bioRxiv a license to display the preprint in perpetuity. It is made available under aCC-BY-NC-ND 4.0 International license.

relationships (b, d, f, and h) for b, Pum Nav1.4 N1609T, d, Hs Nav1.4 N1591T f, Pt Nav1.4 N1600T, and h, Dt Nav1.4 N1600T in presence or absence of $10 \mu \mathrm{M}$ BTX. 


\section{Figure 3}

a<smiles>O1C2OC3OC1OC3O2</smiles>
$500 \mathrm{pA}$ $5 \mathrm{~s}$

C

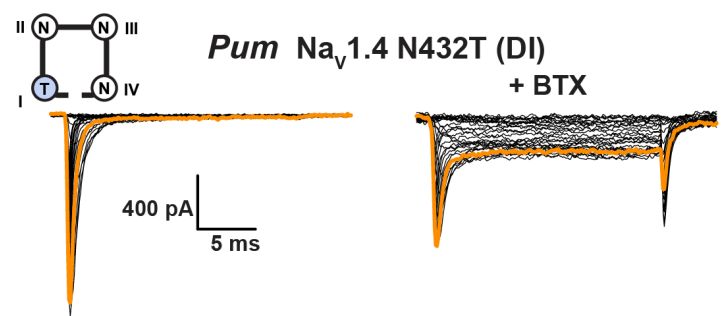

e<smiles></smiles>

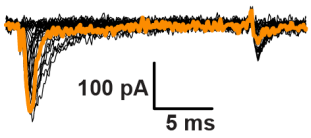

g<smiles></smiles>
$400 \mathrm{pA} \frac{}{5 \mathrm{~ms}}$

Pum $\mathrm{Na}_{\mathrm{v}} 1.4$ N1306T (DIII)

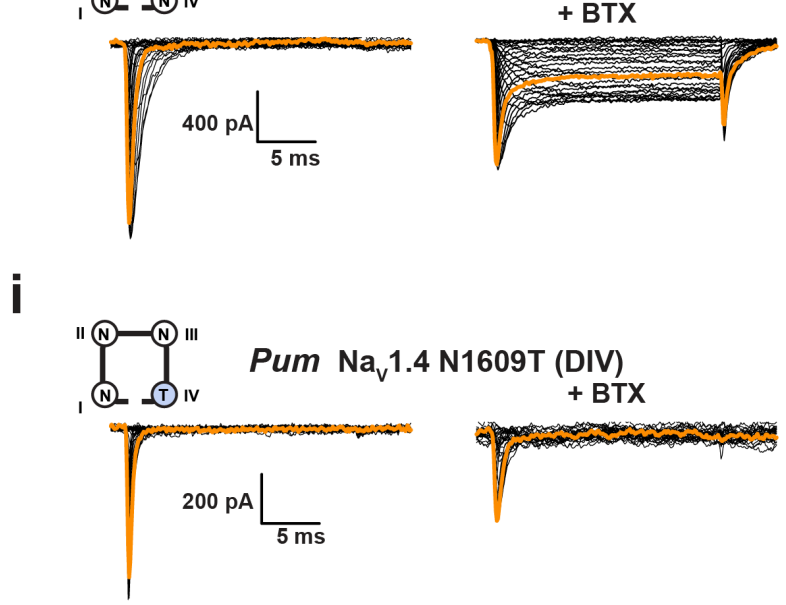

Abderemane-Ali et al.

b

d
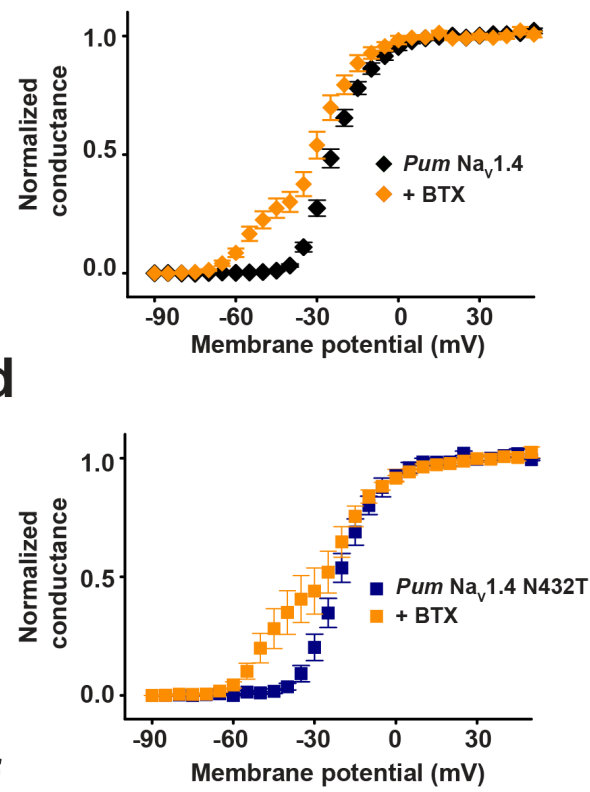

f
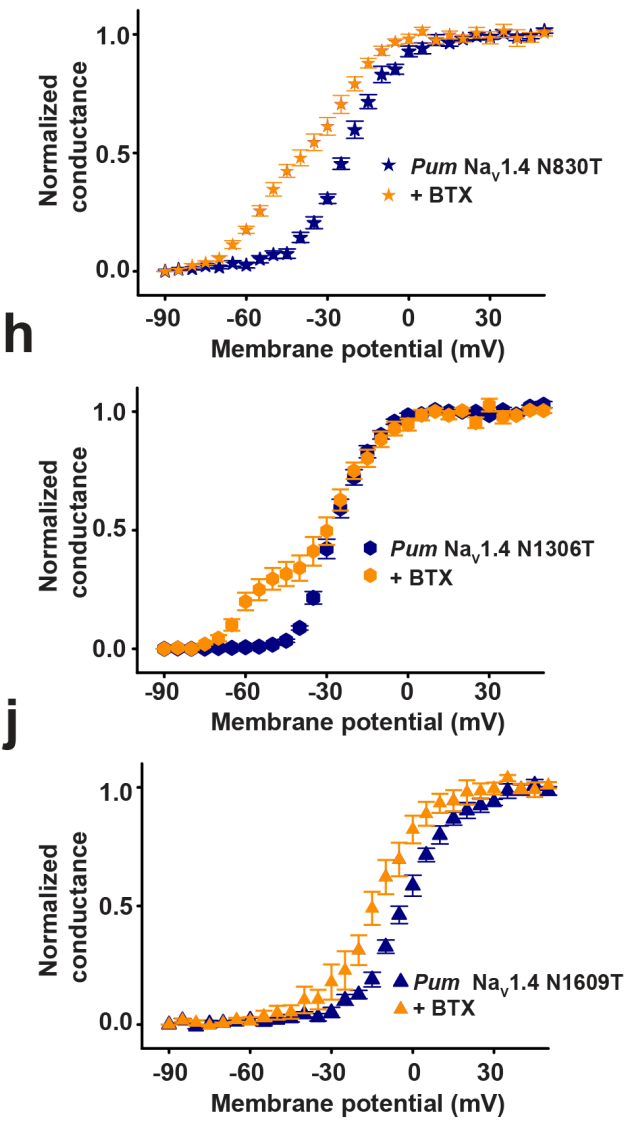

Figure 3. Sodium channel modulation by BTX is associated with an asymmetry at the inner pore level. Exemplar current recordings (a, c, e, g, and i) for: a, Pum Nav1.4; c, Pum Nav1.4 
N432T; e, Pum Nav1.4 N830T ; g, Pum Nav1.4 N1306T, and i, Pum Nav1.4 N1609T expressed in HEK293 cells in absence (left) of presence of $10 \mu \mathrm{M}$ BTX (right). Trace at $0 \mathrm{mV}$ is highlighted in each panel. Currents were evoked with the shown multistep depolarization protocol (inset panel 'a'). Cartoon shows a diagram of the identities of the S6 'Asn' for each construct. Conductance-voltage relationships (b, $\mathbf{d}, \mathbf{f}, \mathbf{h}$, and $\mathbf{j})$ in presence or absence of $10 \mu \mathrm{M}$ BTX for b, Pum Nav1.4 (black diamonds), +BTX (orange diamonds) d, Pum Nav1.4 N432T (dark blue squares), +BTX (orange squares), f, Pum Nav1.4 N830T (dark blue stars), +BTX (orange stars), and h, Pum Nav1.4 N1306T (dark blue hexagons), +BTX (orange hexagons), and j, Pum Nav1.4 N1609T (dark blue triangles), +BTX (orange triangles). Data in ' $a$ ' and 'b' are from Figure 1 a and b. 
Figure 4

a

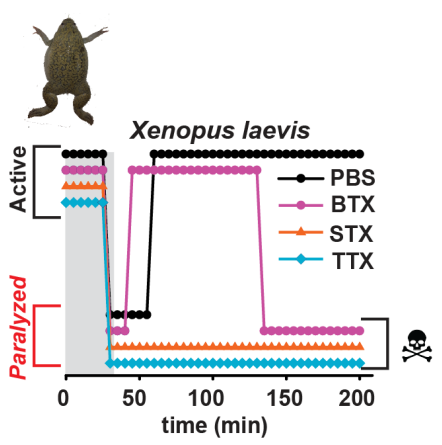

d

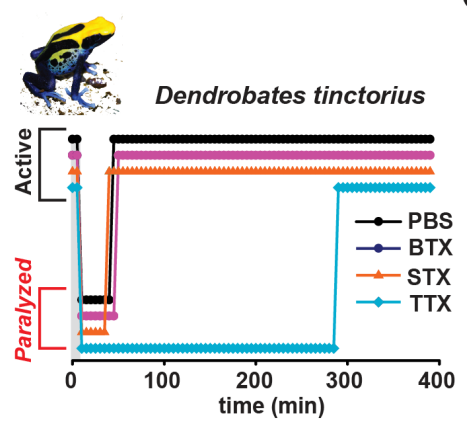

b

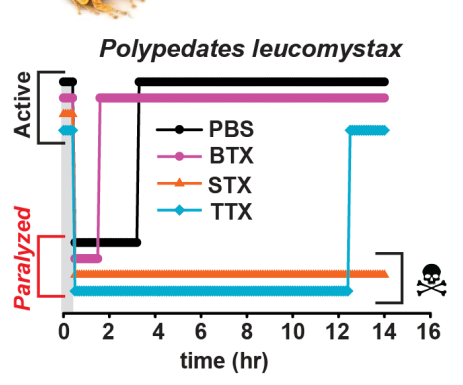

e

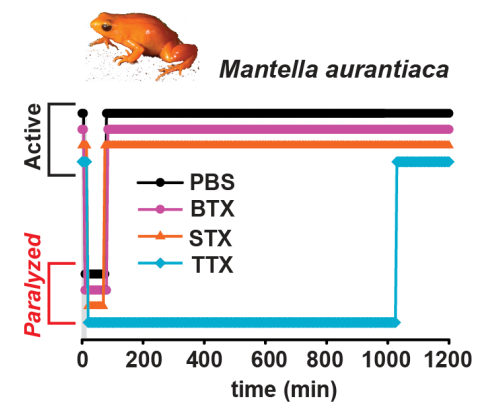

Abderemane-Ali et al.

C

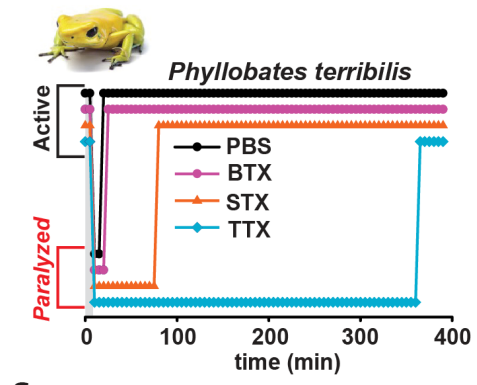

f

\begin{tabular}{|c|c|c|c|}
\cline { 2 - 4 } \multicolumn{1}{l|}{ Frog } & BTX & STX & TTX \\
\hline X. laevis & $\begin{array}{l}\text { Acc. Rec. } \\
\text { Lethal }\end{array}$ & Lethal & Lethal \\
P. leucomystax & Acc. Rec. & Lethal & Resistant \\
P. terribilis & Resistant & Resistant & Resistant \\
D. tinctorius & Resistant & Resistant & Resistant \\
M. aurantiaca & Resistant & Resistant & Resistant
\end{tabular}

Figure 4. Captive-raised poison frogs are resistant to BTX and STX a-f, Challenge experiments for a, Xenopus laevis, b, Polypedates leucomystax, c, Phyllobates terribilis, d, Dendrobates tinctorius, and e, Mantella aurantiaca to PBS (black circles), BTX (magenta circles), STX (orange triangles), or TTX (cyan diamond) injection. Grey area shows period of anesthesia application. Active and paralyzed states of the frogs are indicated. $\mathbf{f}$, Summary of the sensitivity of the indicated species to BTX, STX and TTX. 'Acc. Rec.' denotes accelerated recovery from anesthesia. 'Resistant' denotes no toxin-induced death. 
29 October 2020

List of Supplementary Materials for:

Sodium channel toxin-resistance mutations do not govern batrachotoxin (BTX) autoresistance in poison birds and frogs

Fayal Abderemane-Ali ${ }^{1}$, Nathan D. Rossen ${ }^{1}$, Megan E. Kobiela ${ }^{2}$, Robert A. Craig $\mathrm{II}^{3}$, Catherine E. Garrison $^{3}$, Lauren A. O'Connell ${ }^{4}$, J. Du Bois ${ }^{3}$, John P. Dumbacher ${ }^{5,6}$, and Daniel L. Minor, Jr. ${ }^{1,7-10^{*}}$

Figs. S1-S11

Fig. S1 Pitohui and Poison frog Nav1.4 sequences.

Fig. S2 Pitohui Nav1.5 sequence.

Fig. S3 Pitohui Nav1.5 and Nav1.4:Navß2 complexes are BTX sensitive.

Fig. S4 Structural context for poison frog Navamino acid changes.

Fig. S5 Poison frog Nav1.4s expressed in Xenopus oocytes are BTX sensitive.

Fig. S6 Functional costs of DIV-S6 'Asn' mutation in Rn Nav1.4.

Fig. S7 Functional cost of DIV-S6 'Asn' mutation in Pum Nav1.4 and Hs Nav1.4.

Fig. S8 Functional cost of DIV-S6 $\mathrm{N} \rightarrow \mathrm{T}$ mutation in poison frog Nav1.4s.

Fig. S9 Functional studies of S6 Asn mutants support asymmetric properties of the channel pore.

Fig. S10 BTX competes with anesthetic agent tricaine in Navs from poisonous species.

Fig. S11 Nav1.4s from poisonous animals are STX and TTX sensitive.

\section{Tables S1-S4}

Table S1 $\mathrm{Na}_{v}$ Inactivation parameters.

Table S2 Human $\rightarrow$ Poison frog Nav1.4 amino acid variants.

Table S3 Comparison of the functional effects of S6 mutations.

Table S4 Recovery time from anesthesia.

Gene assembly scripts. 
bioRxiv preprint doi: https://doi.org/10.1101/2020.10.29.361212; this version posted October 29, 2020. The copyright holder for this preprint (which was not certified by peer review) is the author/funder, who has granted bioRxiv a license to display the preprint in perpetuity. It is made available under aCC-BY-NC-ND 4.0 International license.

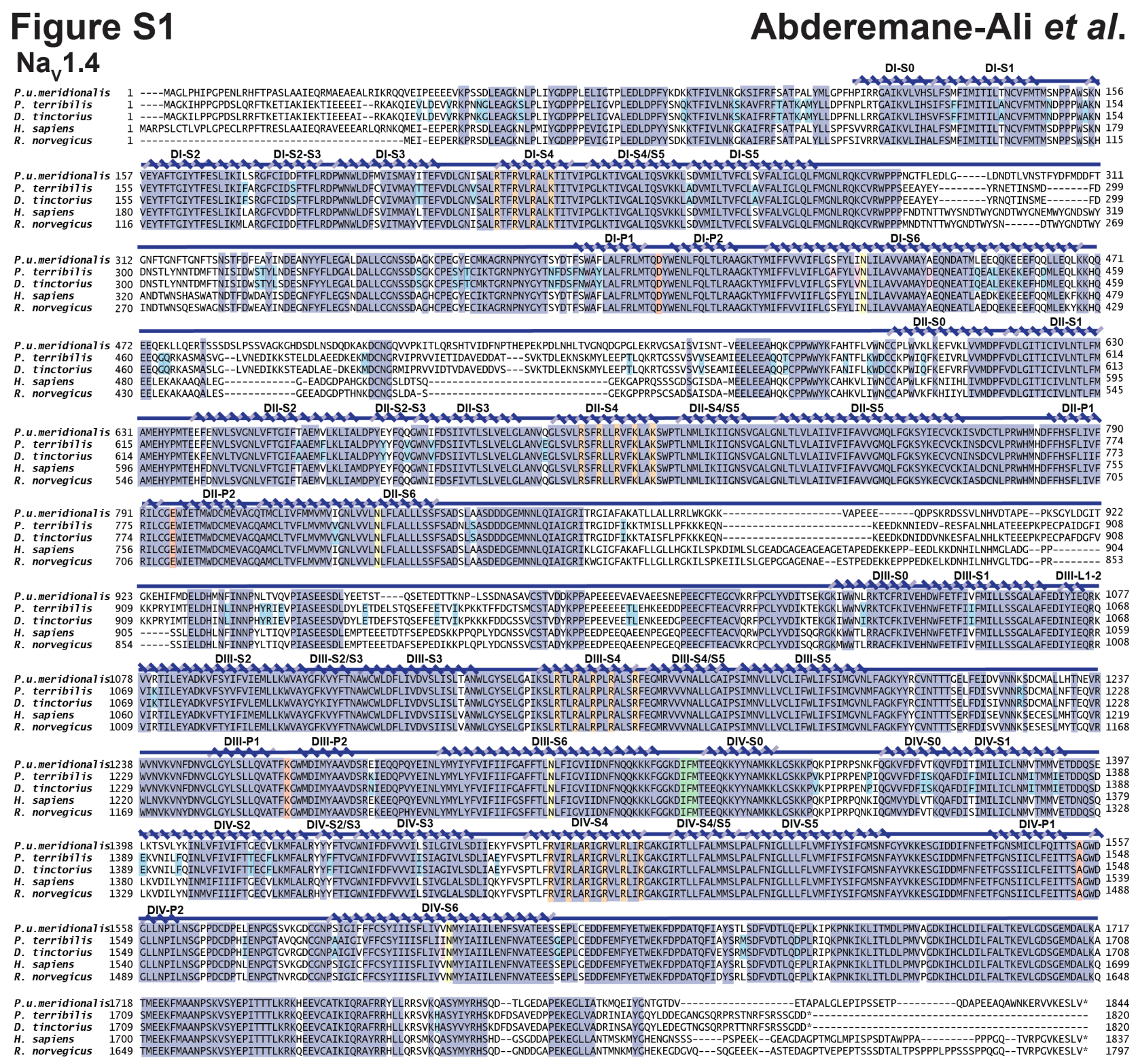

Figure S1 Pitohui and Poison frog Nav1.4 sequences. Sequence alignment of Pitohui uropygialis meridionalis Nav1.4, Phyllobates terribilis Nav1.4, Dendrobates tinctorius Nav1.4, Homo sapiens Nav1.4 (NP_000325.4), and Rattus norgevicus Nav1.4 (NP_037310.1). Key Nav features are highlighted as follows: Selectivity filter 'DEKA' (red), 'IFM' peptide (green), conserved S6 Asn (yellow), S4 voltage sensor arginines (orange), poison frog variants (cyan) and sites highlighted by (24) (magenta) are indicated. Conserved residues are highlighted (dark blue). Secondary structure elements labeled using boundaries from (77). 
bioRxiv preprint doi: https://doi.org/10.1101/2020.10.29.361212; this version posted October 29, 2020. The copyright holder for this preprint (which was not certified by peer review) is the author/funder, who has granted bioRxiv a license to display the preprint in perpetuity. It is made available under aCC-BY-NC-ND 4.0 International license.

29 October 2020

\section{Figure S2}

\section{$\mathrm{Na}_{\mathrm{v}} 1.5$}

P.u.meridionalis $\mathrm{Na}_{1} 1.5$
H. sapiens $\mathrm{Na}, 1.5$

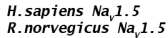

R. norvegicus $\mathrm{Na}_{0} 1.5$
P.u.meridional is $\mathrm{Na}_{\mathrm{t}} 1.4$

P.u.meridionalis $\mathrm{Na}_{\mathrm{1}} 1.5$
H. sapiens Na 159.5

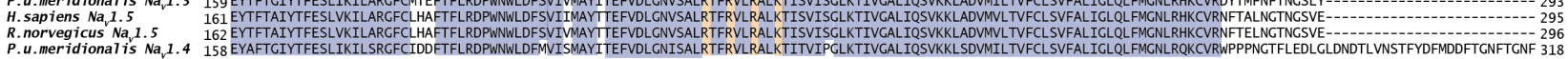

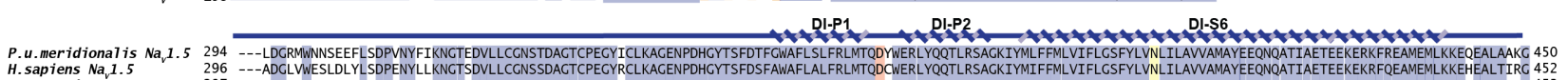

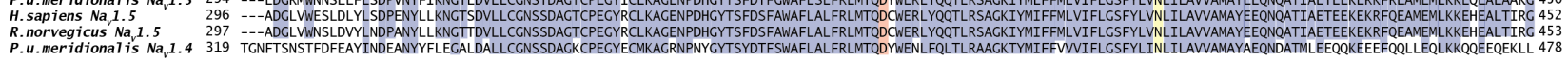

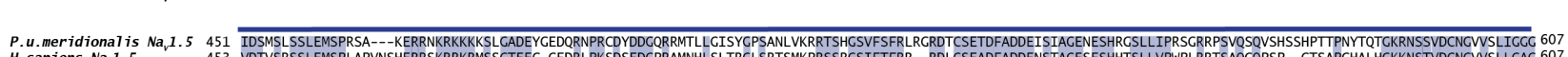

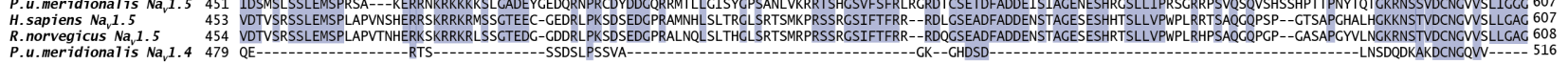

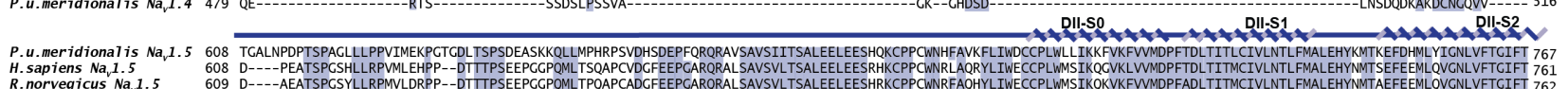

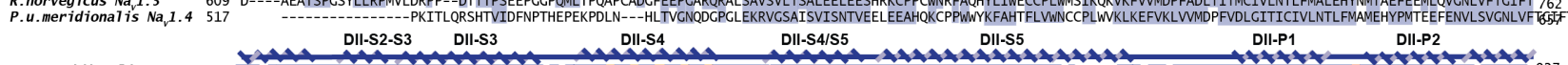

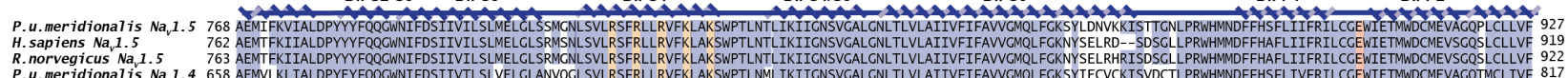

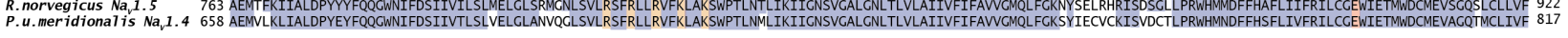
Dll-S6

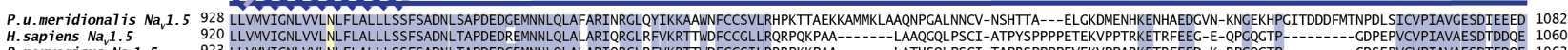

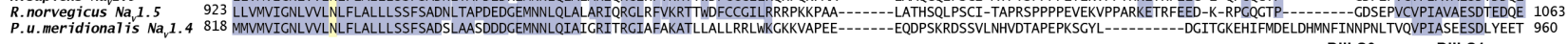

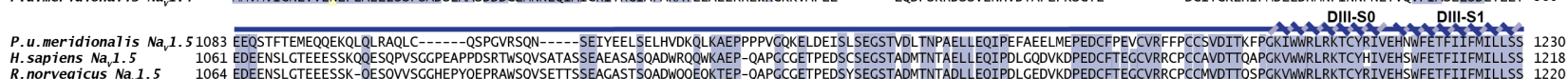

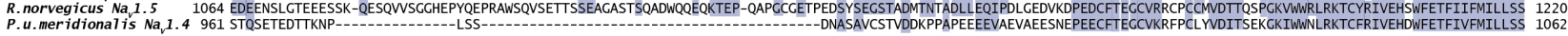

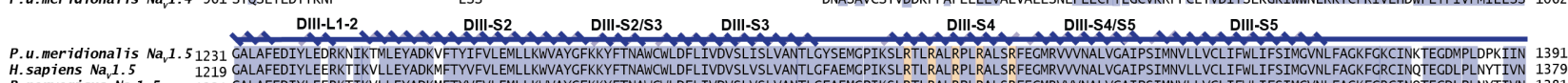

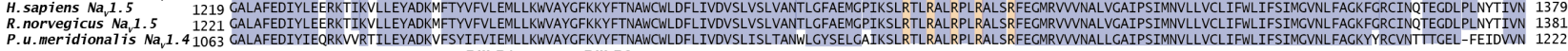

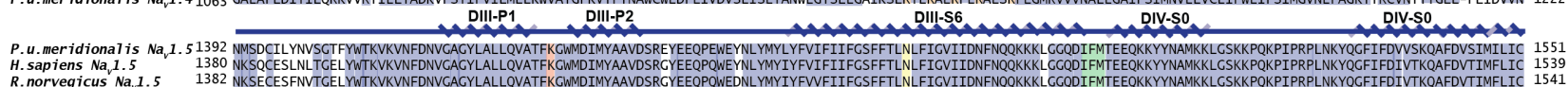

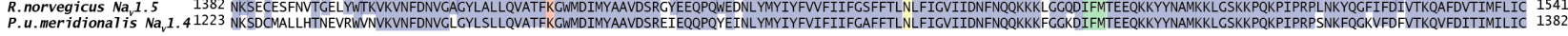

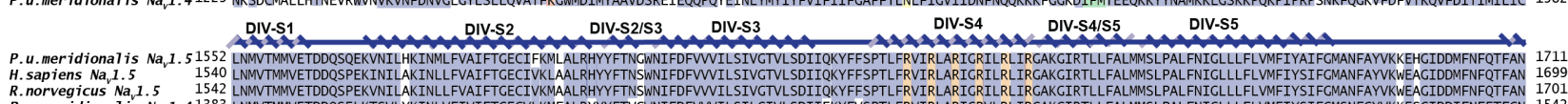

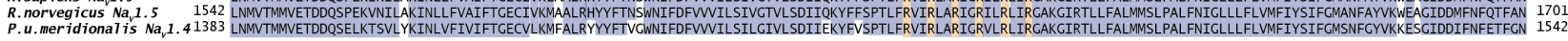

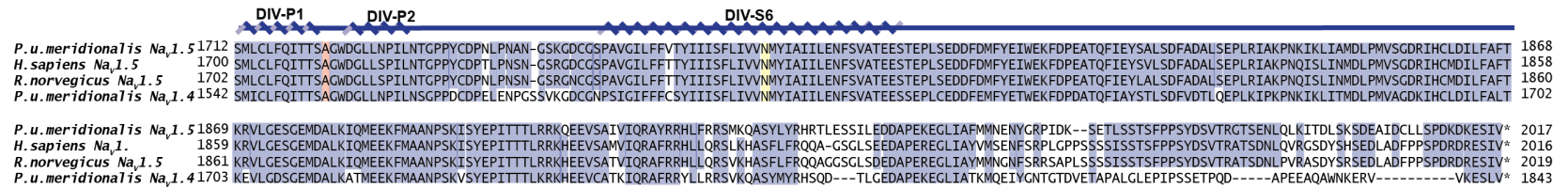

Figure S2 Pitohui Nav1.5 sequence. Sequence alignment of Pitohui uropygialis meridionalis Nav1.5, Homo sapiens Nav1.5 (NP_932173.1), Rattus norgevicus Nav1.5 (NP_037257.1) and Pitohui uropygialis meridionalis $\mathrm{Na} 1.4$. Key $\mathrm{Na}_{\vee}$ features are highlighted as follows: Selectivity filter 'DEKA' (red), 'IFM' peptide (green), conserved S6 Asn (yellow), and S4 voltage sensor arginines (orange). Conserved residues are highlighted (dark blue). Secondary structure elements labeled using boundaries from (77). 
d

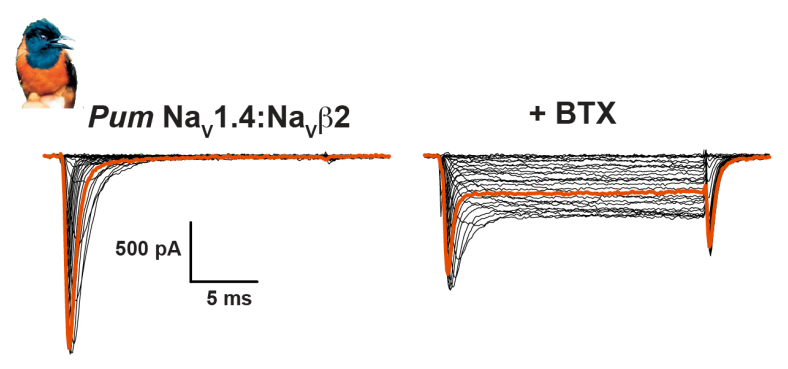

\section{Figure S3}

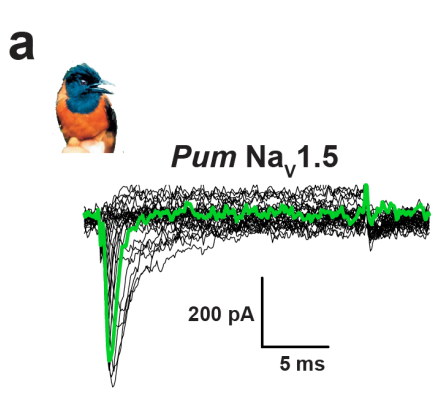

C

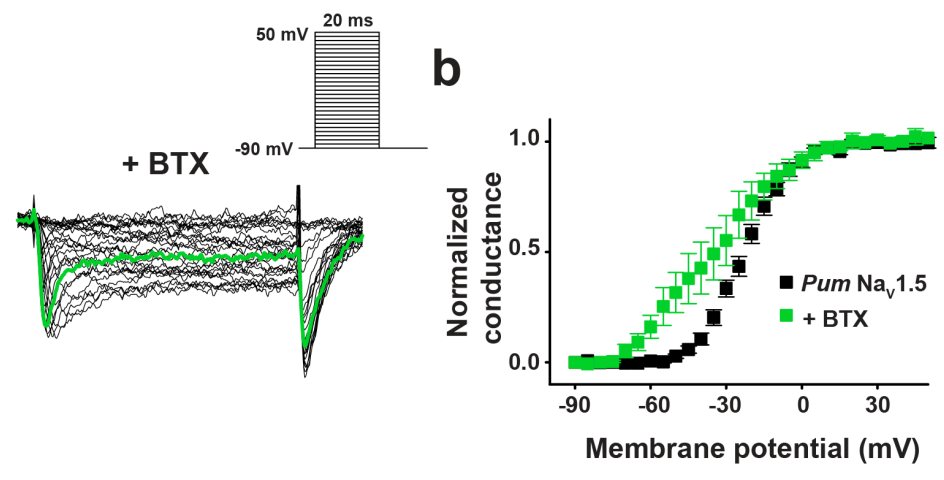

\section{Abderemane-Ali et al.}

$\begin{array}{llll}\beta 1 & \beta 2 & \beta 3 & \beta 4\end{array}$

1 MSPEAWLPQPTLLLTGLTLLLSLAPPGLGMEVMAPATINALNGSSVKLSCTFNSCYKVEN 60 1 MHRDAWLPRPAFSLTGLSLFFSL VPPGRSMEVTVPATLNVLNGSDARLPCTFNSCYTVNH 60 1 MHRDAWLPRPAFSLTGLSLFFSLVPSGRSMEVTVPTTLSVLNGSDTRLPCTFNSCYTVNH 60

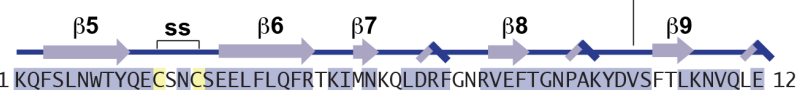

61 KOFSLNWTYOECNNCSEEMFLOFRMKIINLKLERFQDRVEFSGNPSKYDVSVMLRNVQPE 120

61 KQFSLNWTYQECSNCSEEMFLQFRMKIINLKLERFGDRVEFSGNPSKYDVSVTLKNVQLE 120

ss TM

P. u.meridonal is $\mathrm{Na}_{\boldsymbol{\gamma}} \beta 2121$ DEGTYNCYVLNPPDRHRGHGKISLKVLTKEPPKHDSTVAVIVGASVGGFLAVVILVLMVV 180 H. sapiens $\mathrm{Na}_{v} \beta 2$ 121 DEGIYNCYIMNPPDRHRGHGKIHLQVLMEEPPERDSTVAVIVGASVGGFLAVVILVLMVV 180 R.norvegicus $\mathrm{Na}_{v} \beta 2$ 121 DEGIYNCYITNPPDRHRGHGKIYLQVLLEVPPERDSTVAVIVGASVGGFLAVVILVLMVV 180

P. u.meridonal is $N a_{y} \beta 2181$ KCVRRKKQQRLNTDDQKTEEEGKTDGEGNPDEGTK 215

H. sapiens $\mathbf{N a} \beta 2 \quad 181$ KCVRRKKEQKLSTDDLKTEEEGKTDGEGNPDDGAK 215

R. norvegicus $\mathrm{Na}_{v} \beta 2 \quad 181$ KCVRRKKEQKLSTDDLKTEEEGKTDGEGNAEDGAK 215

Figure S3 Pitohui Nav1.5 and Nav1.4:NavB2 complexes are BTX sensitive. a, Exemplar current recordings for Pum Nav1.5 expressed in HEK293 cells in absence (left) or presence of $10 \mu \mathrm{M}$ BTX (right). Trace at $0 \mathrm{mV}$ is highlighted in each panel. Currents were evoked with the shown multistep depolarization protocol (inset). $\mathbf{b}$. Conductance-voltage relationships in absence (black squares) or presence of $10 \mu \mathrm{M}$ BTX (green squares). c, Sequence alignment of Nav $\beta 2$ from Pitohui uropygialis meridionalis, Homo sapiens (NP_004579.1) and Rattus norgevicus (NP_037009.1). Signal peptide (SP), secondary structural elements from (38), conserved disulfide bond (ss), and transmembrane domain (TM) are indicated. d, Exemplar current recordings for Pum Nav1.4:Navß2 expressed in HEK293 cells in absence (left) or presence of 10 $\mu \mathrm{M} B T X$ (right). Trace at $0 \mathrm{mV}$ is highlighted in each panel. Currents were evoked with the shown 
bioRxiv preprint doi: https://doi.org/10.1101/2020.10.29.361212; this version posted October 29, 2020. The copyright holder for this preprint (which was not certified by peer review) is the author/funder, who has granted bioRxiv a license to display the preprint in perpetuity. It is made available under aCC-BY-NC-ND 4.0 International license.

29 October 2020

multistep depolarization protocol (inset panel 'a'). e, Conductance-voltage relationships in absence (black hexagons) or presence of $10 \mu \mathrm{M}$ BTX (red hexagons). 
Figure S4

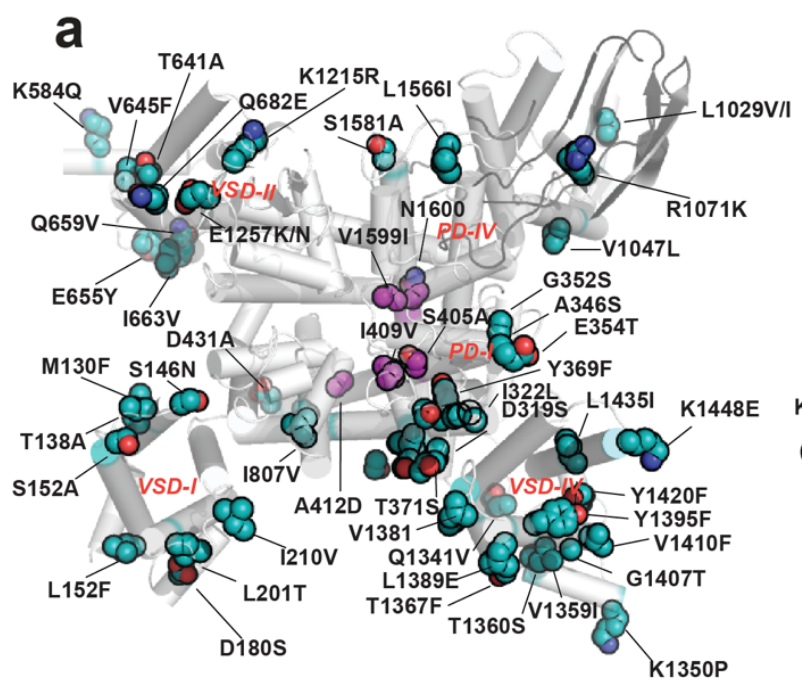

\section{Abderemane-Ali et al.}

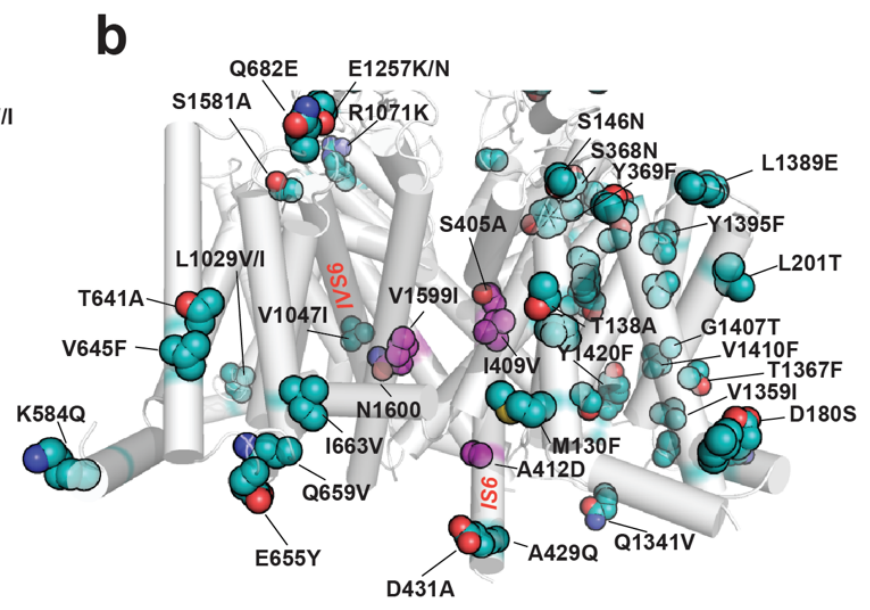

Figure S4 Structural context for poison frog Navamino acid changes. a, and $\mathbf{b}$, Locations of poison frog $\mathrm{Na}_{v}$ amino acid variants reported here (cyan) and shared with (24) (magenta). Variants are denoted human residue:residue number:frog variant using Pt Nav1.4 numbering from Fig. S1. Residues are mapped on human Nav1.4 (PDB:6ADF) (43). Nav1.4 (white). 
Figure S5

a
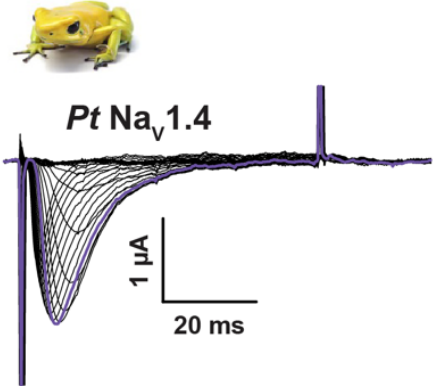

C

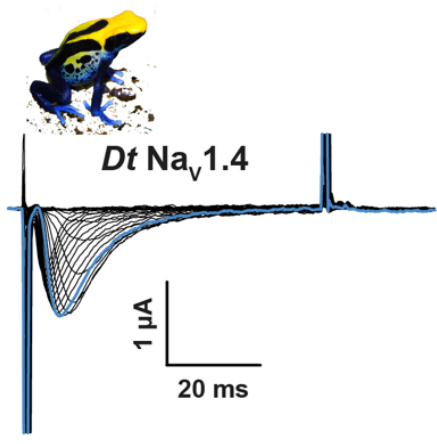

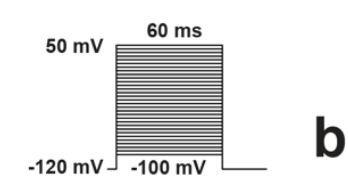
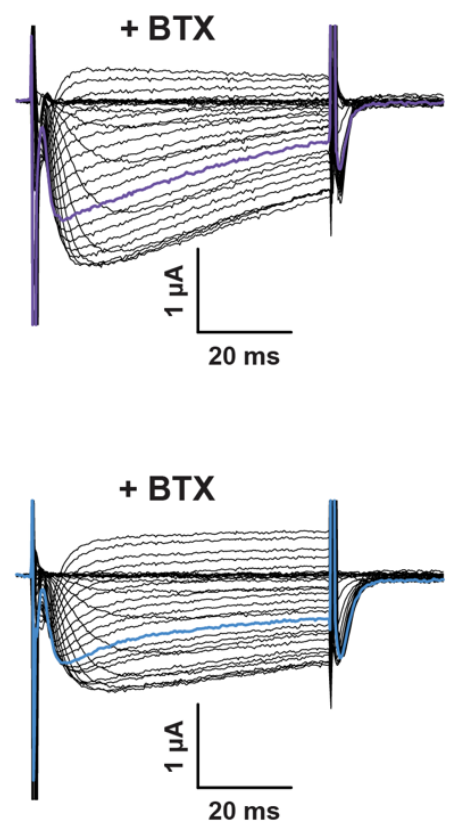

d
Abderemane-Ali et al.
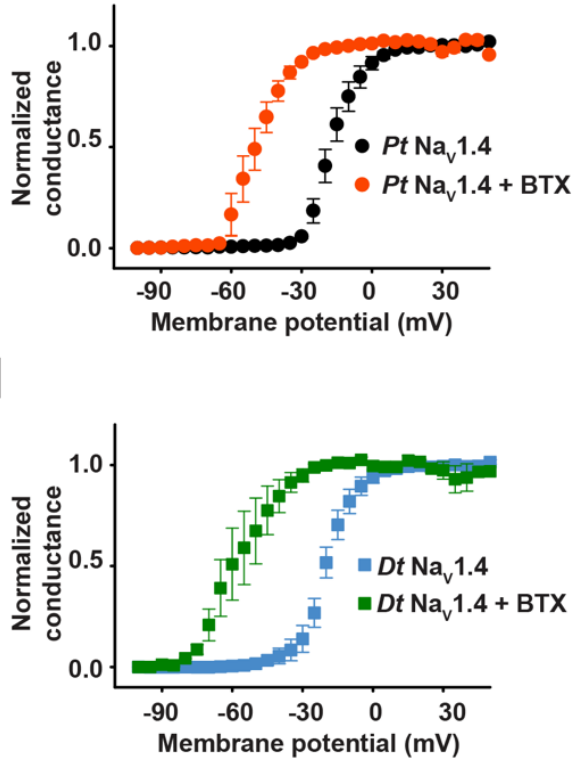

Figure S5 Poison frog Nav1.4s expressed in Xenopus oocytes are BTX sensitive. Exemplar current recordings (a and c) for: a, Phyllobates terribilis Nav1.4 (Pt Nav1.4); and c, Dendrobates tinctorius Nav1.4 (Dt Nav1.4) expressed in Xenopus oocytes in absence (left) or presence of $10 \mu \mathrm{M}$ BTX (right). Trace at $0 \mathrm{mV}$ is highlighted in each panel. Currents were evoked with the shown multistep depolarization protocol (inset panel ' $\mathbf{a}$ '). Conductance-voltage relationships (b and $\mathbf{d}$ ) in presence or absence of $10 \mu \mathrm{M}$ BTX for $\mathbf{b}$, Pt Nav1.4 (black circles), +BTX (orange circles) and d, Dt Nav1.4 (light blue squares), +BTX (green squares). 
Figure S6

a

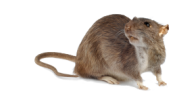

$R n \mathrm{Na}_{\mathrm{v}} 1.4$

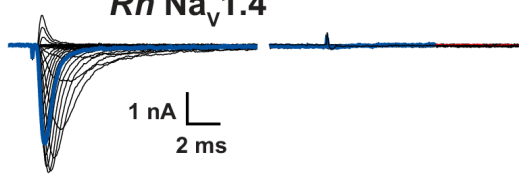

C

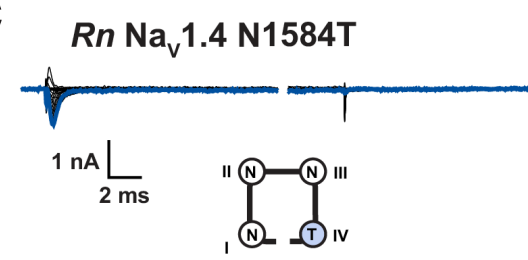

e

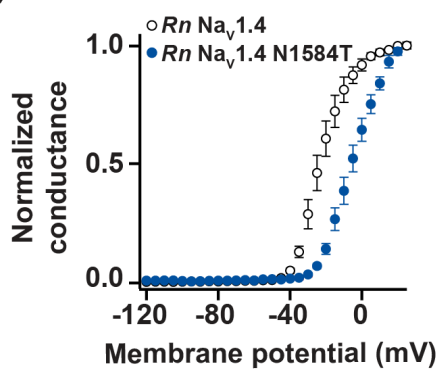

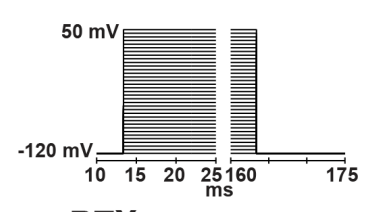

$+\mathrm{BTX}$
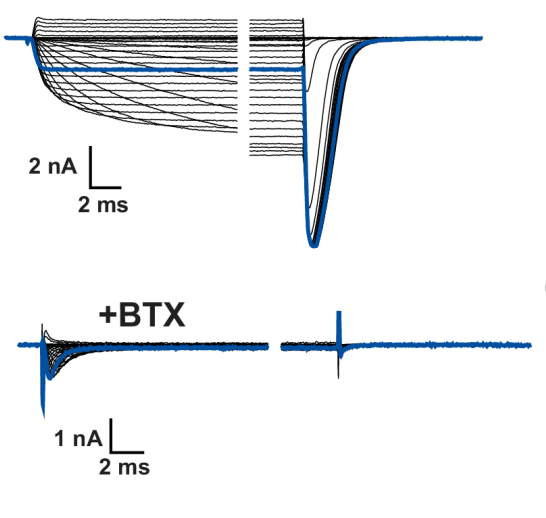

f

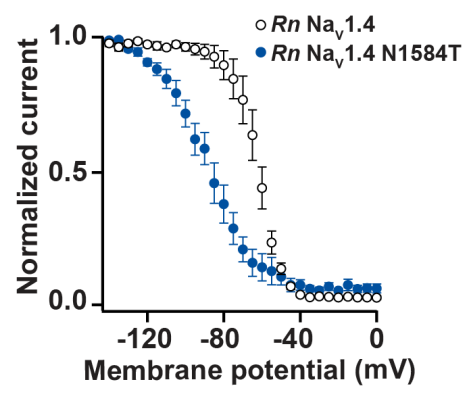

Abderemane-Ali et al.

b

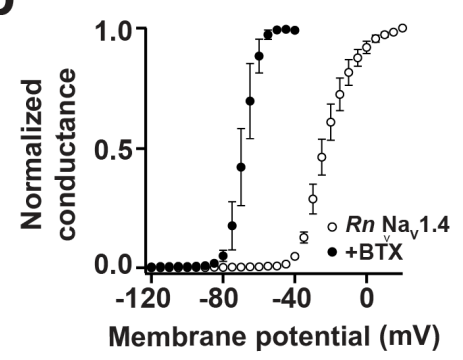

d

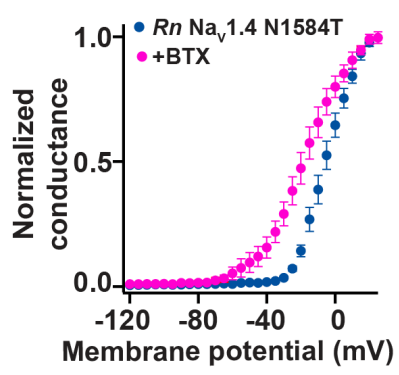

g

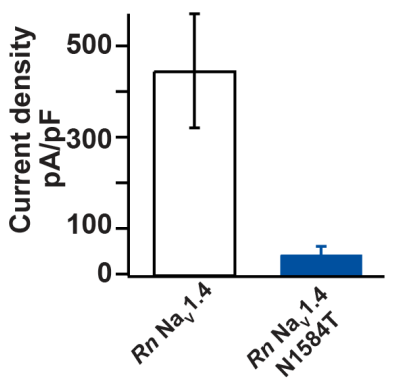

Figure S6 Functional costs of DIV-S6 'Asn' mutation in Rn Nav1.4. Exemplar current recordings (a and c) for: a, $R n$ Nav1.4 and c, $R n$ Nav1.4 N1584T expressed in $\mathrm{CHO}$ cells in absence (left) or presence of $10 \mu \mathrm{M}$ BTX (right). Trace at $0 \mathrm{mV}$ is highlighted in each panel. Currents were evoked with the shown multistep depolarization protocol (inset panel ' $a$ '). Conductance-voltage relationships (b and $\mathbf{d}$ ) in presence or absence of $10 \mu \mathrm{M}$ BTX for b, $R n$ Nav1.4 (open circles), +BTX (black circles) and d, Rn Nav1.4 N1584T (blue circles), +BTX (magenta circles). e, Conductance-voltage relationships, f, Steady-state inactivation voltage dependencies for $R n$ Nav1.4 (open circles) and $R n$ Nav1.4 N1584T (blue circles). g, Current densities for Rn Nav1.4 (white) and Rn Nav1.4 N1584T (blue). 
Figure S7

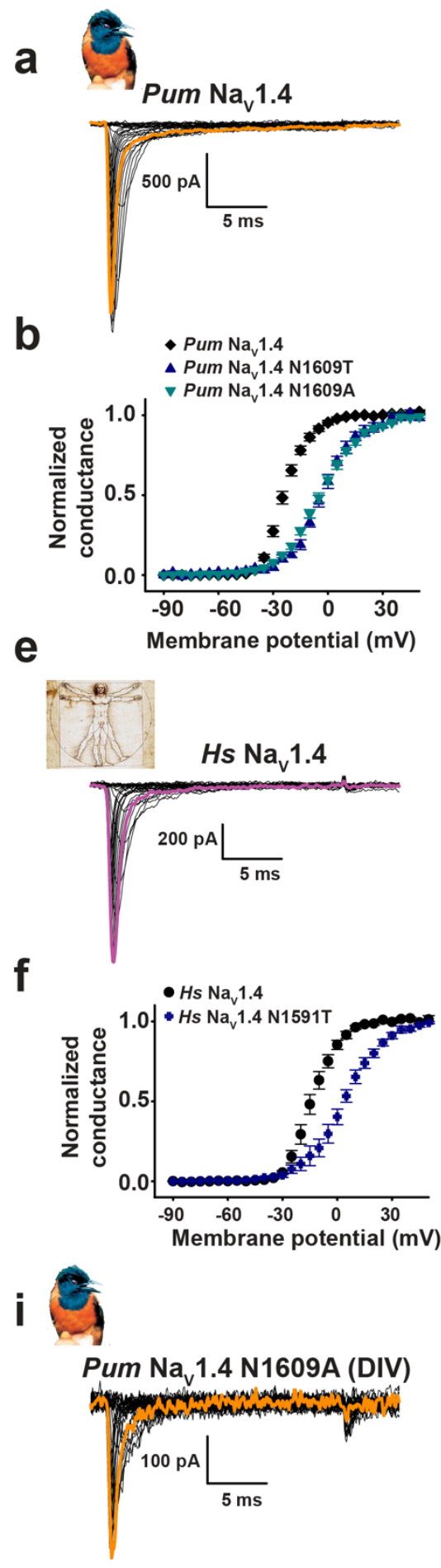

\section{Abderemane-Ali et al.}

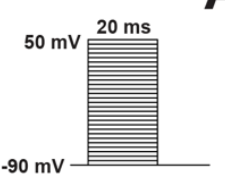

Pum $\mathrm{Na}_{\mathrm{v}} 1.4$ N1609T (DIV)

Pum $\mathrm{Na}_{\mathrm{v}} 1.4$ N1609A (DIV)

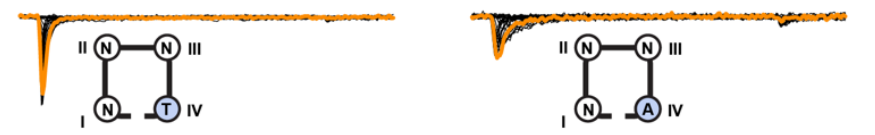

C
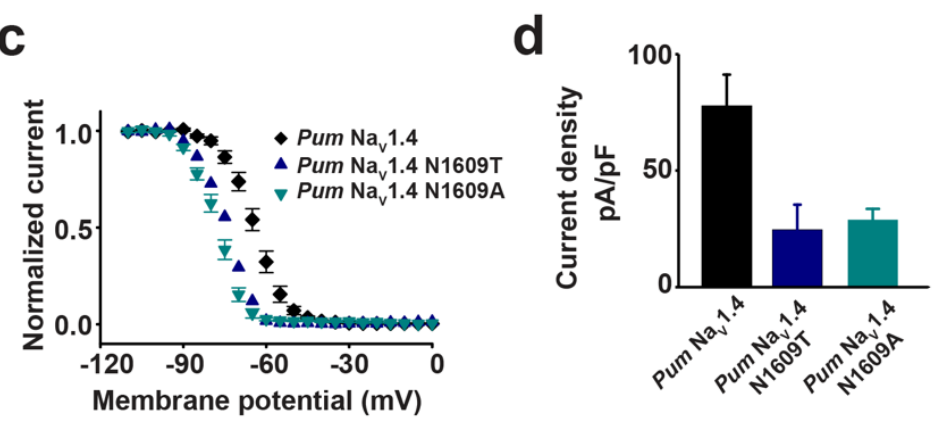

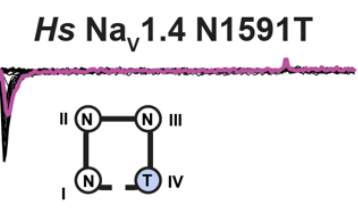

g

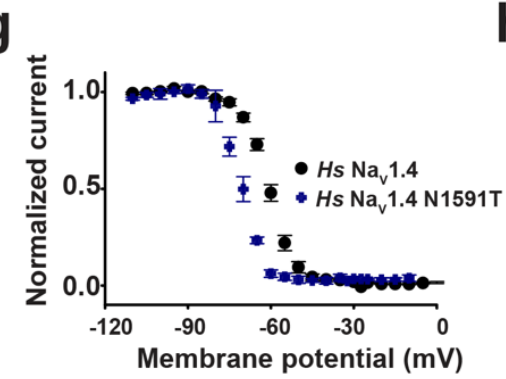

h

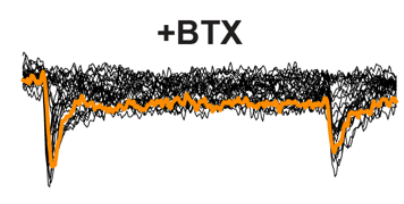

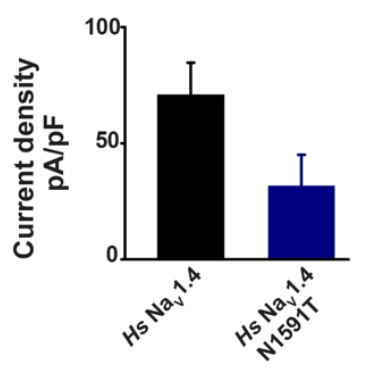

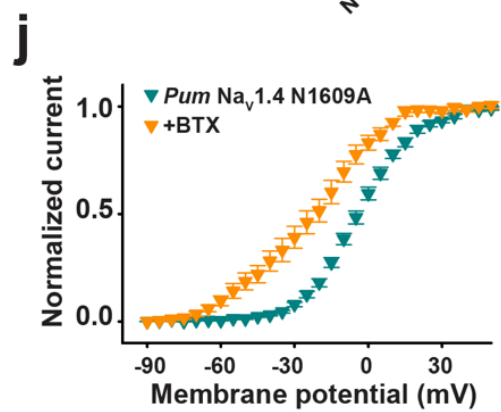

Figure S7 Functional cost of DIV-S6 'Asn' mutation in Pum Nav1.4 and Hs Nav1.4. Exemplar current recordings (for: a, Pum Nav1.4 (left), Pum Nav1.4 N1609T (middle), and Pum Nav1.4 
N1609A (right) expressed in HEK293 cells. Trace at $0 \mathrm{mV}$ is highlighted and currents were evoked with the shown multistep depolarization protocol (inset). Cartoon shows a diagram of the identities of the S6 'Asn' for the 'Asn' mutants. b, Conductance-voltage relationships, c, steady-state inactivation voltage dependencies and d, current densities for Pum Nav1.4 (black diamonds), Pum Nav1.4 N1609T (blue triangles), and Pum Nav1.4 N1609A (teal inverted triangles). e, Exemplar current recordings for $\mathrm{Hs}$ Nav1.4 (left), Hs Nav1.4 N1591T (right), expressed in HEK293 cells. Trace at $0 \mathrm{mV}$ is highlighted. Currents were evoked with the shown multistep depolarization protocol from ' $a$ '. $\mathbf{f}$, Conductance-voltage relationships, $\mathbf{g}$, steady-state inactivation voltage dependencies and $\mathbf{h}$, current densities $H s$ Nav1.4 (black circles) and Hs Nav1.4 N1591T (blue diamonds). i, Exemplar current recordings for Pum Nav1.4 N1609A (left) and in the presence of $10 \mu \mathrm{M}$ BTX (right). j, Conductance-voltage relationships for Pum Nav1.4 N1609A (green inverted triangles) and in the presence of $10 \mu \mathrm{M} \mathrm{BTX}$ (orange inverted triangles). 
Figure S8

a

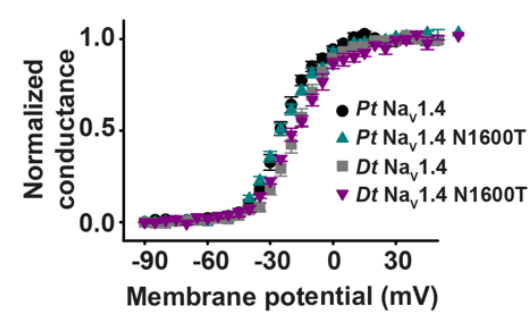

d

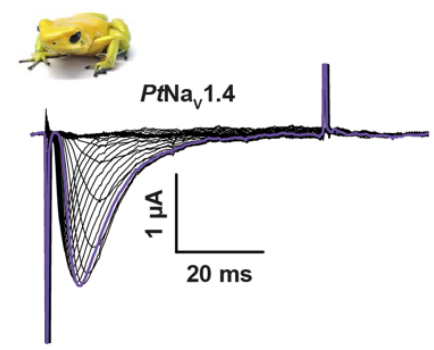

f

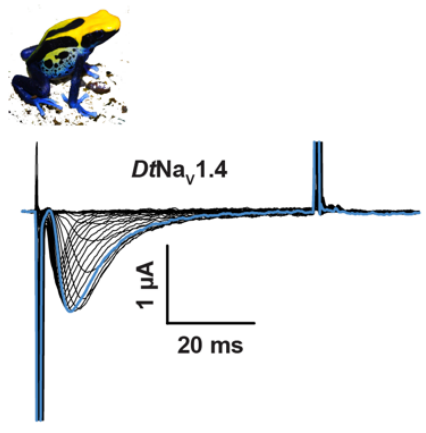

h

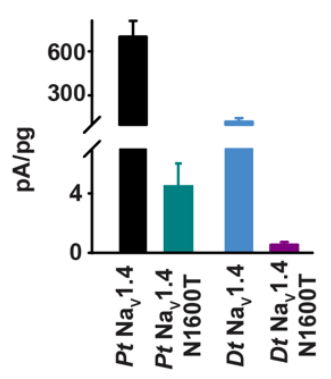

b

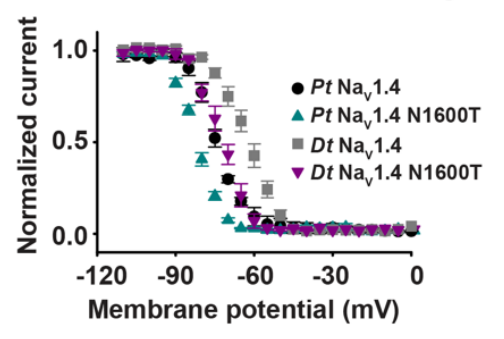

e

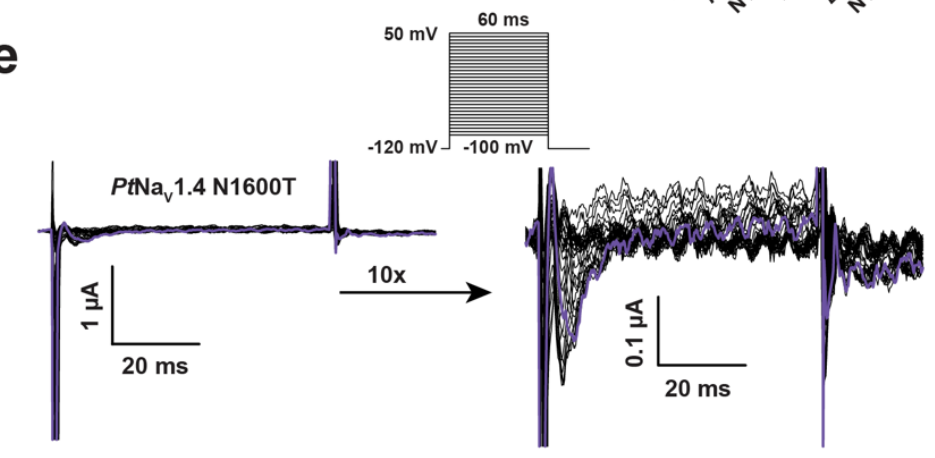

g

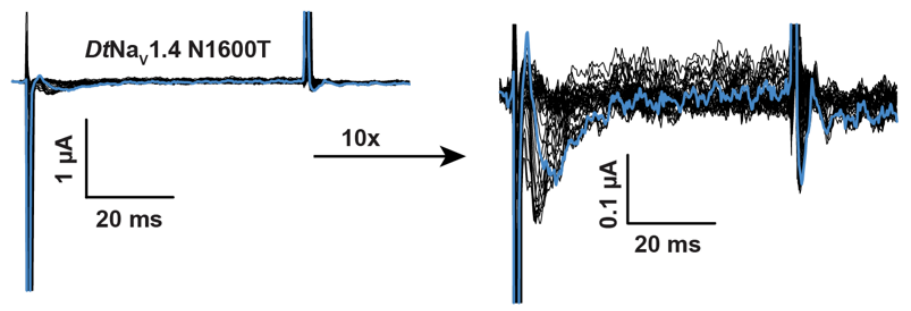

Figure S8 Functional cost of DIV-S6 $\mathrm{N} \rightarrow \mathrm{T}$ mutation in poison frog Nav1.4s. a, Conductance-voltage relationships, b. Steady-state inactivation voltage dependences, and c, current densities for Pt Nav1.4, Pt Nav1.4 N1600T, Dt Nav1.4, and Dt Nav1.4 N1600T expressed in HEK293 cells. Exemplar current recordings (d, e, f, and g) for: d, Pt Nav1.4, e, Pt Nav1.4 N1600T, f, Dt Nav1.4, and g, Dt Nav1.4 N1600T expressed in Xenopus oocytes. 10 times magnification of Pt Nav1.4 N1600T and Dt Nav1.4 N1600T traces are shown (e and $\mathbf{g}$, right panels). Trace at $0 \mathrm{mV}$ is highlighted in each panel. Currents were evoked with the shown multistep depolarization protocol (inset panel 'e'). h, Current amplitudes normalized to the amount of injected RNA for the indicated poison frog constructs. 
Figure S9

a

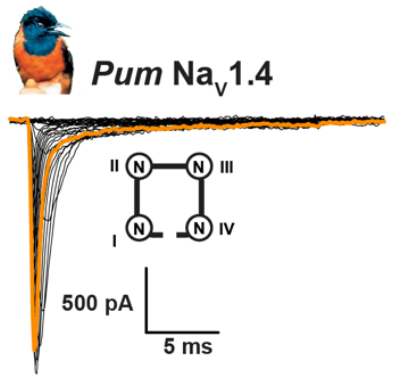

Pum Na 1.4 N1306T (DIII)

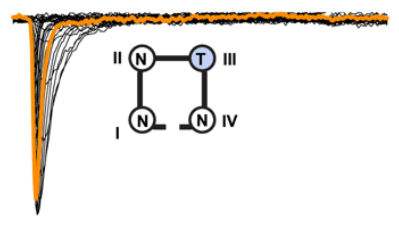

b

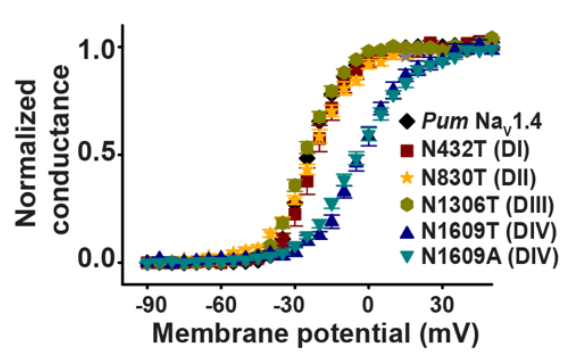

e

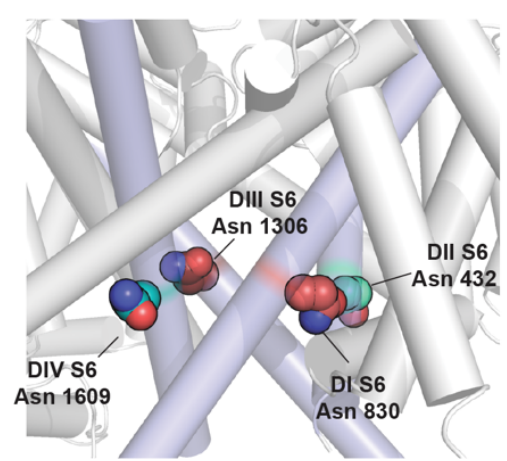

\section{Abderemane-Ali et al.}
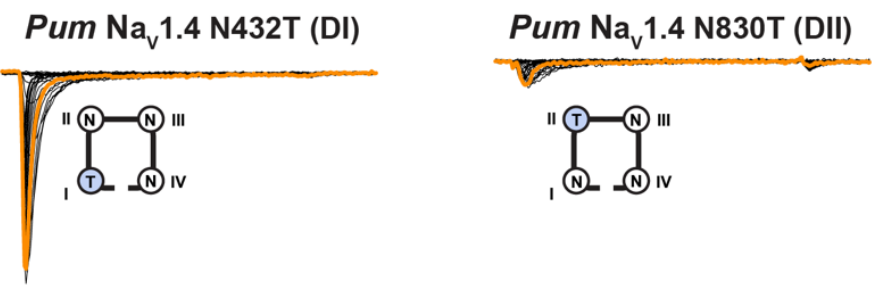

Pum $\mathrm{Na}_{\mathrm{v}} 1.4$ N1609T (DIV)

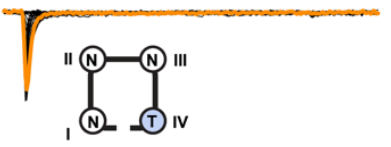

Pum $\mathrm{Na}_{\mathrm{v}} 1.4$ N1609A (DIV)

C

d
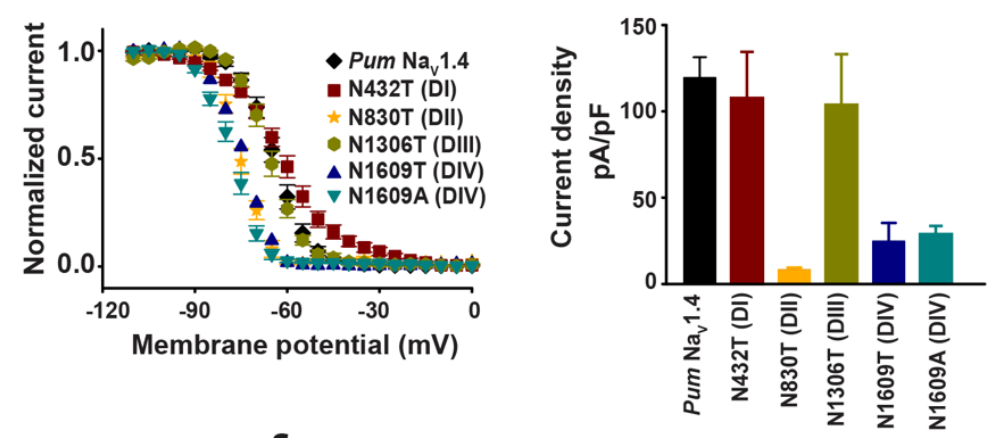

f

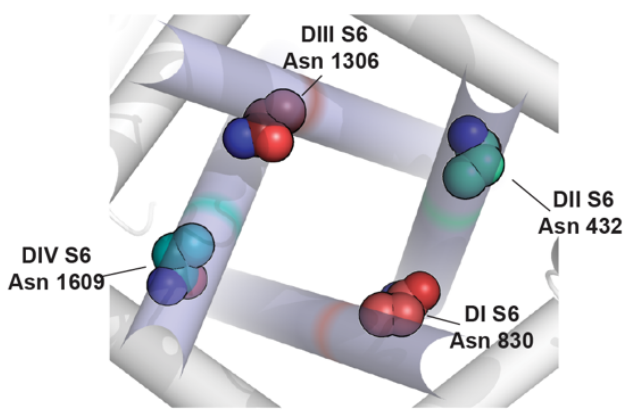

Figure S9 Functional studies of S6 Asn mutants support asymmetric properties of the channel pore. a, Exemplar current recordings, b, conductance-voltage relationships, c, steady-state inactivation voltage dependences, and d, current densities for Pum Nav1.4, Pum Nav1.4 N432T, Pum Nav1.4 N830T, Pum Nav1.4 N1306T, Pum Nav1.4 N1609T, and Pum Nav1.4 $\mathrm{N} 1609 \mathrm{~A}$ expressed in HEK293 cells. Trace at $0 \mathrm{mV}$ is highlighted in each panel. Cartoon shows a diagram of the identities of the S6 'Asn' for each construct. e, and f, Side 'e' and bottom ' $f$ ' views of the locations the $\mathrm{S} 6$ conserved asparagines. Residues are mapped on the structure of human Nav1.4 (PDB:6ADF) (43) and are labeled using the Pum Nav1.4 numbering. 
Figure S10

Abderemane-Ali et al.

a

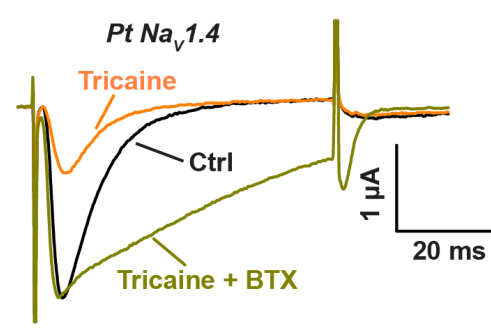

d

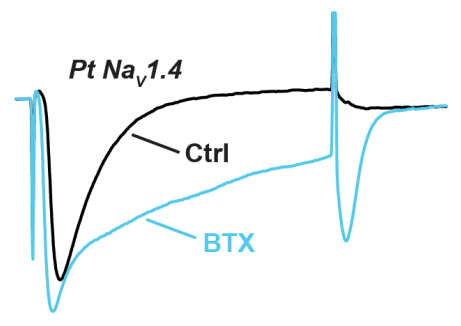

g

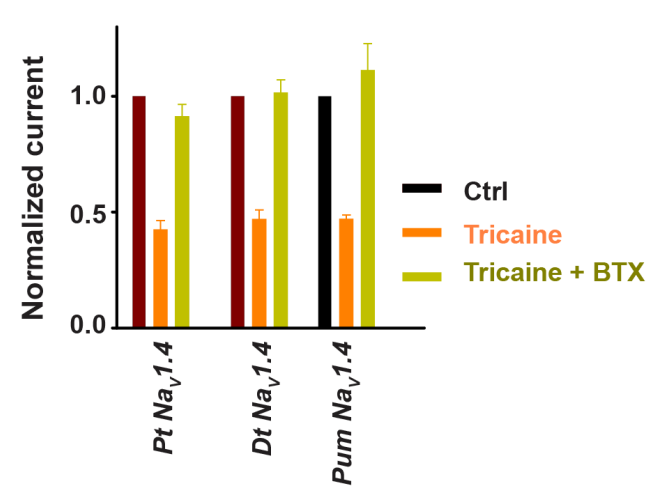

b

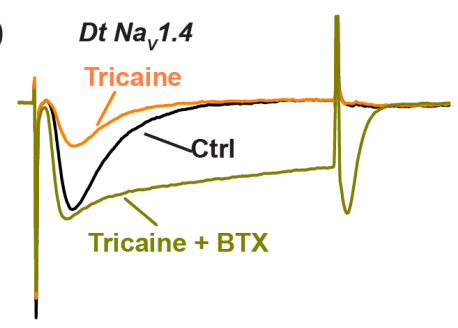

e

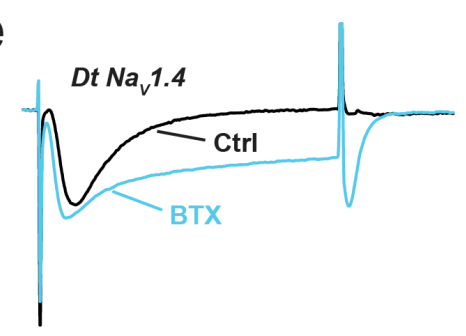

C Pum $\mathrm{Na}_{\mathrm{v}} 1.4$

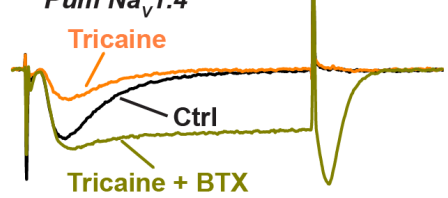

h

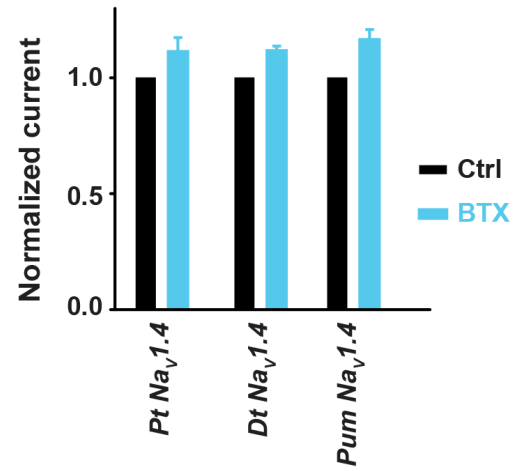

Figure S10 BTX competes with anesthetic agent tricaine in Navs from poisonous species. a, Exemplar recordings at $0 \mathrm{mV}$ in control (black), after $0.5 \mathrm{mM}$ tricaine application (orange) and after BTX injection (dark green) into the same Xenopus oocytes expressing Pt Nav1.4 (left), Dt Nav1.4 (middle) or Pum Nav1.4 (right). BTX injection was performed after tricaine block of sodium current and the recordings of the BTX effect were done while the oocyte was still exposed to tricaine. b. Exemplar recordings at $0 \mathrm{mV}$ before (black) or after BTX injection (light blue) into the same Xenopus oocytes expressing Nav1.4 from the indicated poisonous species. c and d, Average peak current amplitudes normalized to the corresponding control peak current amplitude. 


\section{Figure S11}

a

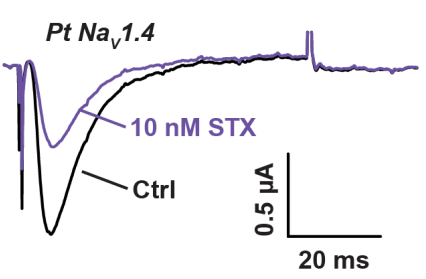

C

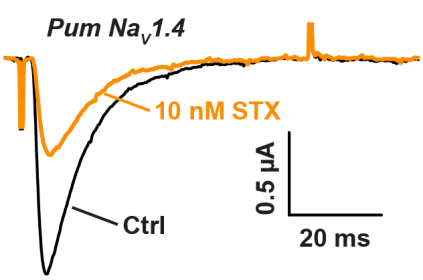

e

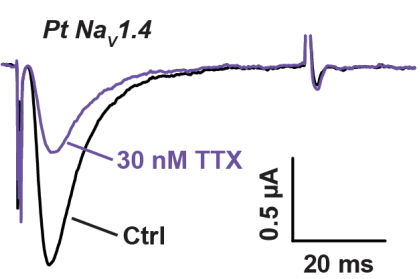

g

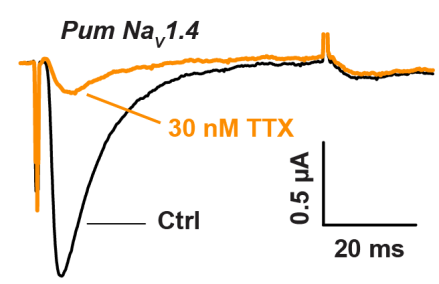

\section{Abderemane-Ali et al.}

b
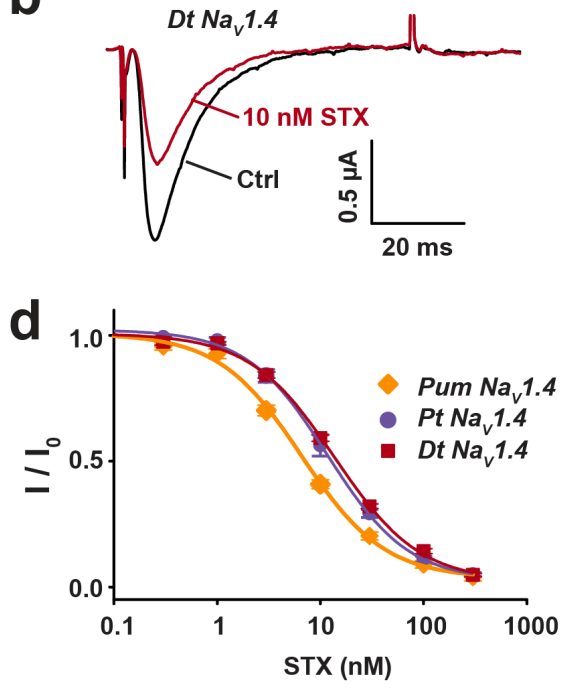

f

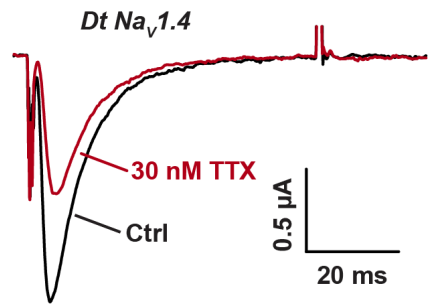

h

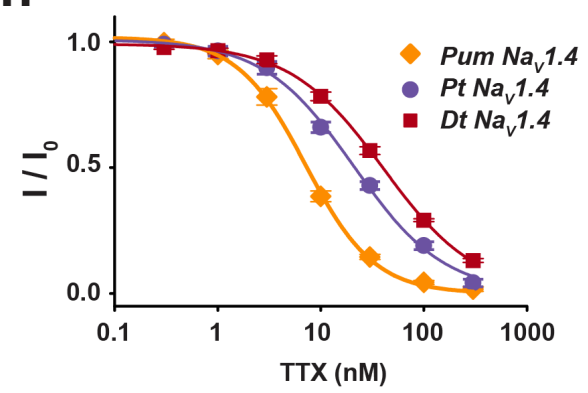

Figure S11 Nav1.4s from poisonous animals are STX and TTX sensitive. a-c, Exemplar recordings at $0 \mathrm{mV}$ before (black) and after $10 \mathrm{nM}$ STX application to Xenopus oocytes expressing a, Pt Nav1.4 (purple) b, Dt Nav1.4 (red), or c, Pum Nav1.4 (orange). d, STX Dose-response curves for Pt Nav1.4 (purple circles), Dt Nav1.4 (red squares), and Pum Nav1.4 (orange diamonds). Curves show fits to the Hill equation. ( $\mathrm{IC}_{50}=12.6 \pm 1.4 \mathrm{nM}, 14.6 \pm 0.6 \mathrm{nM}$, and $7.3 \pm 0.5 \mathrm{nM}$ for Pt Nav1.4, Dt Nav1.4, and Pum Nav1.4, respectively. Errors are SEM. $(\mathrm{n}=4)$. e-g, Exemplar recordings at $0 \mathrm{mV}$ before (black) and after $30 \mathrm{nM}$ TTX application to Xenopus oocytes expressing e, Pt Nav1.4 (purple), f, Dt Nav1.4 (red), or g, Pum Nav1.4 (orange). h, TTX Dose-response curves for Pt Nav1.4 (purple circles), Dt Nav1.4 (red squares), and Pum Nav1.4 (orange diamonds). Curves show fits to the Hill equation. $\left(\mathrm{IC}_{50}=21.3 \pm 1.0 \mathrm{nM}, 40.8 \pm 1.8 \mathrm{nM}\right.$, and $6.2 \pm 0.4 \mathrm{nM}$ for Pt Nav1.4, Dt Nav1.4, and Pum Nav1.4, respectively. Errors are SEM. $(n=5-6)$. 
bioRxiv preprint doi: https://doi.org/10.1101/2020.10.29.361212; this version posted October 29, 2020. The copyright holder for this preprint (which was not certified by peer review) is the author/funder, who has granted bioRxiv a license to display the preprint in perpetuity. It is made available under aCC-BY-NC-ND 4.0 International license.

29 October 2020

Table S1 $\mathrm{Na}_{v}$ Inactivation parameters

\begin{tabular}{|c|c|c|c|c|c|}
\hline & Channel & $\mathrm{V}_{1 / 2 \text { inact }}$ & $\Delta \mathrm{V}_{1 / 2}$ inact & $\mathrm{k}_{\text {inact }}$ & $\mathrm{n}$ \\
\hline \multirow{8}{*}{ ?. } & Pum Nav1.4 & $-64.2 \pm 1.3$ & n.a. & $4.8 \pm 0.1$ & 14 \\
\hline & Pum Nav1.4 + PkNav $\beta 2$ & $-66.5 \pm 1.4$ & n.a. & $4.6 \pm 0.2$ & 8 \\
\hline & Pum Nav1.5 & $-75.9 \pm 1.6$ & n.a. & $4.2 \pm 0.5$ & 6 \\
\hline & Pum Nav1.4 N432T (DI) & $-61.2 \pm 1.6$ & n.a. & $9.8 \pm 0.5$ & 6 \\
\hline & Pum Nav1.4 N830T (DII) & $-75.0 \pm 0.9$ & $-10.8 \pm 1.6$ & $4.2 \pm 0.2$ & 7 \\
\hline & Pum Nav1.4 N1306T (DIII) & $-65.3 \pm 1.0$ & n.a. & $5.1 \pm 0.5$ & 7 \\
\hline & Pum Nav1.4 N1609T (DIV) & $-74.5 \pm 1.7$ & $-10.3 \pm 2.1$ & $4.9 \pm 0.1$ & 3 \\
\hline & Pum Nav1.4 N1609A (DIV) & $-77.6 \pm 0.9$ & $-13.4 \pm 1.6$ & $4.7 \pm 0.2$ & 10 \\
\hline \multirow{3}{*}{$\begin{array}{l}\mathbf{\Upsilon} \\
\mathbf{\Xi} \\
\mathbf{1}\end{array}$} & Hs Nav1.4 & $-60.5 \pm 0.8$ & n.a. & $4.7 \pm 0.3$ & 12 \\
\hline & Hs Nav1.4 N1591T (DIV) & $-70.3 \pm 0.9$ & $-9.8 \pm 1.2$ & $4.2 \pm 0.2$ & 3 \\
\hline & Rn Nav1.4 & $-61.9 \pm 0.2$ & n.a. & $6.1 \pm 0.2$ & 4 \\
\hline$\frac{\pi}{\pi}$ & Rn Nav1.4 N1584T (DIV) & $-88.9 \pm 0.4$ & $-27.0 \pm 0.4$ & $12.6 \pm 0.4$ & 6 \\
\hline \multirow{4}{*}{$\begin{array}{l}8 \\
0 \\
\delta \\
0 \\
0 \\
0\end{array}$} & Pt Nav1.4 & $-73.9 \pm 0.6$ & n.a. & $5.9 \pm 1.4$ & 3 \\
\hline & Pt Nav1.4 (N1600T) (DIV) & $-81.6 \pm 0.7$ & $-7.7 \pm 0.9$ & $4.7 \pm 0.3$ & 6 \\
\hline & $D t \mathrm{Na}_{\mathrm{v}} 1.4$ & $-62.2 \pm 1.3$ & n.a. & $5.6 \pm 0.5$ & 8 \\
\hline & Dt Nav1.4 (N1600T) (DIV) & $-72.0 \pm 1.4$ & $-9.8 \pm 1.9$ & $5.2 \pm 0.3$ & 3 \\
\hline
\end{tabular}


bioRxiv preprint doi: https://doi.org/10.1101/2020.10.29.361212; this version posted October 29,2020 . The copyright holder for this preprint (which was not certified by peer review) is the author/funder, who has granted bioRxiv a license to display the preprint in perpetuity. It is made available under aCC-BY-NC-ND 4.0 International license.

29 October 2020

Table S2 Human $\rightarrow$ Poison frog Nav1.4 amino acid variants

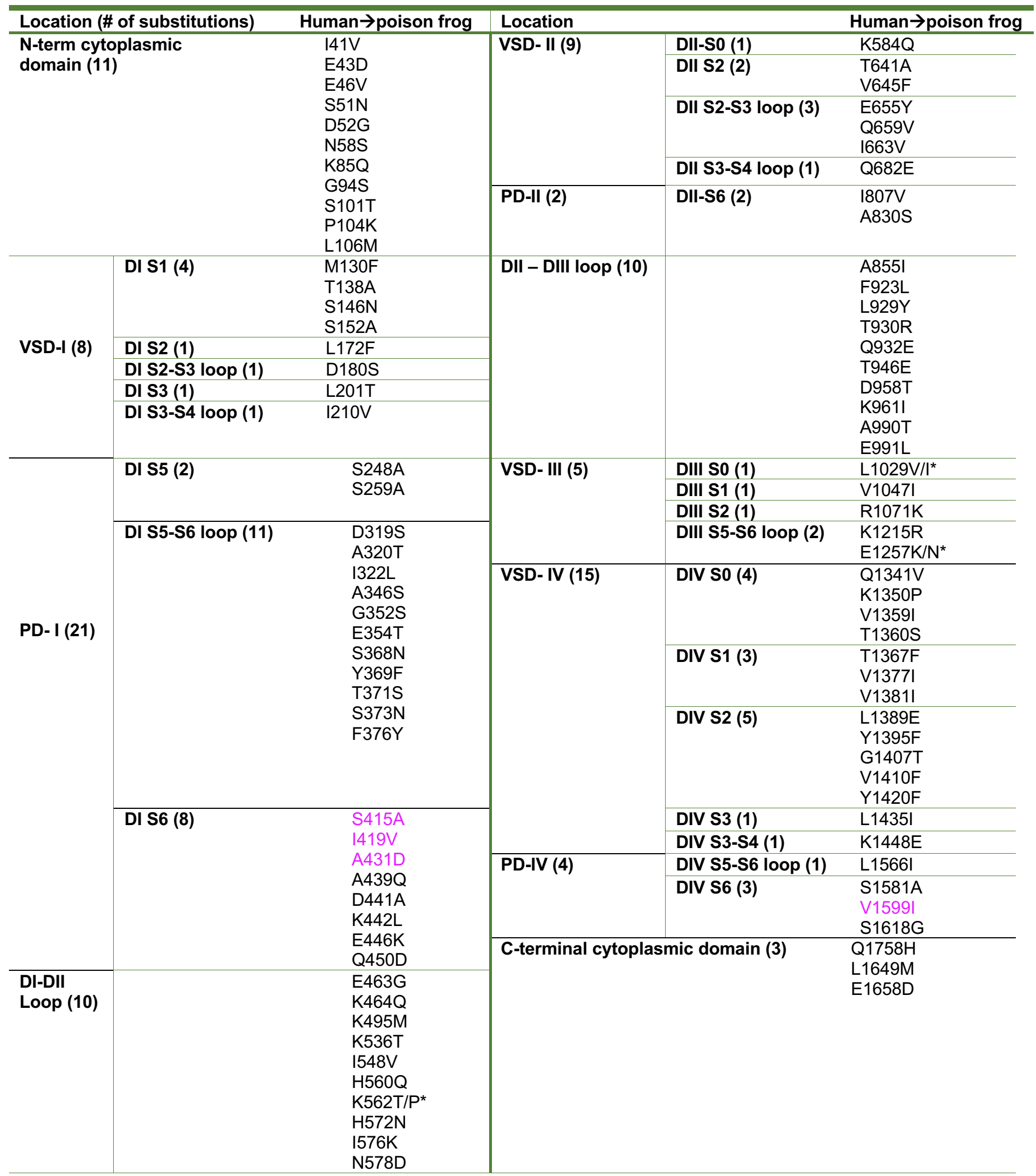

Pore domain (PD) and voltage sensor domain (VSD) are indicated.

Magenta residues are variants shared with (24). 
bioRxiv preprint doi: https://doi.org/10.1101/2020.10.29.361212; this version posted October 29, 2020. The copyright holder for this preprint (which was not certified by peer review) is the author/funder, who has granted bioRxiv a license to display the preprint in perpetuity. It is made available under aCC-BY-NC-ND 4.0 International license.

\section{October 2020}

Variations are denoted using the human residue identity, Pt Nav1.4 number, and poison frog residue identity. * indicates site where Pt Nav1.4 and Dt Nav1.4 differ. 
bioRxiv preprint doi: https://doi.org/10.1101/2020.10.29.361212; this version posted October 29, 2020. The copyright holder for this preprint (which was not certified by peer review) is the author/funder, who has granted bioRxiv a license to display the preprint in perpetuity. It is made available under aCC-BY-NC-ND 4.0 International license.

29 October 2020

Table S3 Comparison of the functional effects of S6 mutations

\begin{tabular}{lllll}
\hline Channel & $\begin{array}{l}\text { BTX } \\
\text { resistant? }\end{array}$ & $\begin{array}{l}\text { Activation } \\
\text { shift? } \\
\Delta \mathbf{V}_{1 / 2} \text { act }(\mathrm{mV})\end{array}$ & $\begin{array}{l}\text { Inactivation } \\
\text { shift? } \\
\Delta \mathbf{V}_{1 / 2} \text { inact }(\mathrm{mV})\end{array}$ & $\begin{array}{l}\text { Decrease in } \\
\text { current density? } \\
\text { (fold change) }\end{array}$ \\
\hline Pum Nav1.4 N432T (DI) & No & No & No & No \\
Pum Nav1.4 N830T (DII) & No & No & Yes; $-10.8 \pm 1.6$ & Yes; 14.8 \\
Pum Nav1.4 N1306T (DIII) & No & No & No & No \\
Pum Nav1.4 N1609T (DIV) & Yes & Yes; +20.5 \pm 1.5 & Yes; $-10.3 \pm 2.1$ & Yes; 4.9 \\
Pum Nav1.4 N1609A (DIV) & No & Yes; +19.6 \pm 1.6 & Yes; $-13.4 \pm 1.6$ & Yes; 4.1 \\
Hs Nav1.4 N1591T (DIV) & Yes & Yes; +16.9 \pm 2.7 & Yes; $-9.8 \pm 1.2$ & Yes; 2.2 \\
\hline Rn Nav1.4 N1584T (DIV) & Yes & Yes; +18.0 \pm 0.6 & Yes; $-27.0 \pm 0.4$ & Yes; 10.3 \\
Pt Nav1.4 (N1600T) (DIV) & No & No & Yes; $-7.7 \pm 0.9$ & No* \\
Dt Nav1.4 (N1600T) (DIV) & No & No & Yes; -9.8 \pm 1.9 & No* \\
\hline
\end{tabular}

Data represent measurements in transfected HEK293 cells

‘*' indicates substantial current density loss in Xenopus oocytes. 
bioRxiv preprint doi: https://doi.org/10.1101/2020.10.29.361212; this version posted October 29,2020 . The copyright holder for this preprint (which was not certified by peer review) is the author/funder, who has granted bioRxiv a license to display the preprint in perpetuity. It is made available under aCC-BY-NC-ND 4.0 International license.

29 October 2020

Table S4 Recovery time from anesthesia (minutes)

\begin{tabular}{lllll}
\hline & PBS & BTX & STX & TTX \\
\hline X. laevis & $29 \pm 1$ & $15 \pm 2$ & N/A & N/A \\
P.leucomystax & $169 \pm 12$ & $70 \pm 20$ & N/A & $720 \pm 30$ \\
P. terribilis & $10 \pm 1$ & $14 \pm 2$ & $72 \pm 8$ & $354 \pm 6$ \\
D. tinctorius & $35 \pm 4$ & $38 \pm 3$ & $32 \pm 14$ & $283 \pm 57$ \\
M. aurantiaca & $56 \pm 11$ & $60 \pm 10$ & $52 \pm 3$ & $1011 \pm 51$
\end{tabular}

Values are average \pm S.E.M. 
bioRxiv preprint doi: https://doi.org/10.1101/2020.10.29.361212; this version posted October 29, 2020. The copyright holder for this preprint (which was not certified by peer review) is the author/funder, who has granted bioRxiv a license to display the preprint in perpetuity. It is made available under aCC-BY-NC-ND 4.0 International license.

\title{
29 October 2020
}

\section{Gene assembly scripts.}

\author{
$\# ! / \mathrm{bin} / \mathrm{sh}$
}

\#this script should search for reads matching sodium channels, create a seed file from MiSeq and HiSeq reads, and start a PRICE assembly of the sodium channel using these seeds

\#these steps use blatq to search for reads matching the sodium channel echo "\$d"_starting blatq search to pull seeds for PRICE

blatq -t=dna /data/jdumbacher/Pitohui_Nav/BlastLibs/SCN4A-Corvus_cornixGenomic.fa

/data/jdumbacher/Pitohui_Nextera/Pitohui_HiSeq_data/Final_Fastq/Pitohui157 HiSeq_ALLDATA.trimmed.fästq

/data/jumbacher/Pitohui_Nav/BlastResults/SCN4A-

Corvus_cornix_Hi.Seq_ALLDĀTA_matches.m8 -out=blast8

blatq -t=dna /data/jdumbacher/Pitohui_Nav/BlastLibs/SCN4A-Corvus_cornixGenomic.fa

/data/jdumbacher/Pitohui_Nextera/Pitohui_MiSeq_data/Final_BBMerged_Trimmed Files/Pitohui157_MiSeq_ĀLLDATA_trimmed.'̄fastq

Tdata/jdumbacher/Pitohui_Nav/BlastResults/SCN4A-

Corvus_cornix_MiSeq_ALLDĀTA_matches.m8 -out=blast8

echo "\$Q" Blatq done

\# Step 2 exerpt reads by id:

echo "\$d"Now starting to excerpt reads with blatq hits by ID

/data/jdumbacher/bin/excerptByIDs

/data/jdumbacher/Pitohui_Nav/BlastResults/SCN4A-

Corvus_cornix_HiSeq_ALLDĀTA_matches.m8

/data/joumbacher/Pitohui_Nextera/Pitohui_HiSeq_data/Final_Fastq/Pitohui157 HiSeq_ALLDATA.trimmed.fastq

/data/jdumbacher/Pitohui_Nav/BlastResults/SCN4A-

Corvus_cornix_HiSeq_ALLDĀTA_matches.fastq

/data/jdumbacher/bin/excerptByIDs

/data/jdumbacher/Pitohui_Nav/BlastResults/SCN4A-

Corvus_cornix_MiSeq_ALLDĀTA_matches.m8

/data/joumbacher/Pitohui_Nextera/Pitohui_MiSeq_data/Final_BBMerged_Trimmed Files/Pitohui157_MiSeq_ĀLLDATA_trimmed. fastq

Tdata/jdumbacher/Pitohui_Nav/BlastResults/SCN4A-

Corvus_cornix_MiSeq_ALLDĀTA_matches.fastq

echo "\$d"done excerpting reads

cat

/data/jdumbacher/Pitohui_Nav/BlastResults/SCN4A-

Corvus_cornix_HiSeq_ALLDATA_matches.fastq

/data/jumbacher/Pitohui_Nā̄/BlastResults/SCN4A-

Corvus_cornix_MiSeq_ALLDĀTA_matches.fastq

/data/jombacher/Pitohui_Nav/BlastResults/SCN4A-Corvus_cornix.seeds.fastq 
bioRxiv preprint doi: https://doi.org/10.1101/2020.10.29.361212; this version posted October 29, 2020. The copyright holder for this preprint (which was not certified by peer review) is the author/funder, who has granted bioRxiv a license to display the preprint in perpetuity. It is made available under aCC-BY-NC-ND 4.0 International license.

\title{
29 October 2020
}

\author{
echo "\$Q"number of seeds in seed file, \\ /data/jdumbacher/Pitohui_Nav/BlastResults/SCN4A-Corvus_cornix.seeds.fastq \\ grep -c" "c" /data/jdumbacher/Pitohui_Nav/BlastResults/sCN4A- \\ Corvus_cornix.seeds.fastq \\ \# for larger read sets, assemble initial reads using spades: \\ python /data/jdumbacher/SPAdes-3.9.0-Linux/bin/spades.py --careful -s \\ /data/jdumbacher/Pitohui_Nav/BlastResults/SCN4A-Corvus_cornix.seeds.fastq \\ -o - /data/jdumbacher/Pitohui_Nav/BlastResults/SCN4A- \\ Corvus_cornix.assembled-seeds \\ \# Assemble using PRICE (For PRICE assemblies - use note for PRICE \\ assemblies Labnotes 25 November 2014) \\ echo "\$Q"Now starting PRICE assembly using reads \\ PriceTI \\ /data/jdumbacher/Pitohui_Nextera/Pitohui_MiSeq_data/Final_BBMerged_Trimmed \\ _Files/Pitohui157_Run1_BB̈Unmerged_trimmed_paired_R1.fastq \\ Tdata/jdumbacher/Pitohüi_Nextera/Pitohui_ĒiSeq_dāta/Final_BBMerged_Trimmed

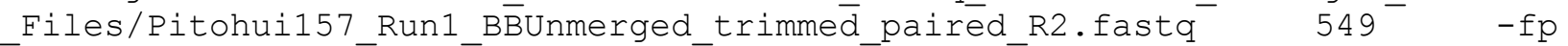 \\ T/data/jdumbacher/Pitohüi_Nextera/Pitohui_HiSeq_dāta/Final_Fastq/Pitohui157 \\ Hiseq PE trimmed R1.fastq \\ Tdata/joumbacher/Pitohui_Nextera/Pitohui_HiSeq_data/Final_Fastq/Pitohui157 \\ HiSeq_PE_trimmed R2.fastq $\quad-\quad 482 \quad-i c f$ \\ Tdata/joumbacher/Pitohui_Nav/BlastResults/SCN4A-Corvus_cornix.assembled- \\ seeds/contigs.fasta 1 I $\overline{5}$-mol 30 -mpi 90 -MPI 85 -nc 50 -a 10 -target 85

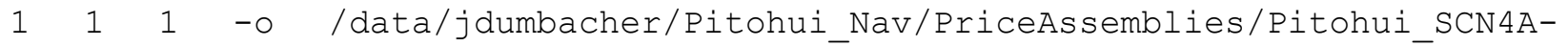 \\ from_Corvus_cornix.Ass-seeds.fa \\ /datā/jdumbācher/Pitohui_Nav/PriceAssemblies/Pitohui_SCN4A- \\ from_Corvus_cornix.ass-seeds.priceq $\quad$-maxHp - $25 \quad-$ logf \\ /datājoumbācher/Pitohui_Nav/PriceAssemblies/Pitohui_SCN4A- \\ from_Corvus_cornix.ass-sēeds.log \\ /datājoumbācher/Pitohui_Nav/PriceAssemblies/Pitohui_SCN4A- \\ from_Corvus_cornix.ass-seeds.errorlog
}

